\title{
A higher order compact finite difference algorithm for solving the incompressible Navier-Stokes equations
}

\author{
Zhenfu Tian ${ }^{1, *, \dagger}$, Xian Liang ${ }^{2}$ and Peixiang $\mathrm{Yu}^{1}$ \\ ${ }^{1}$ Department of Mechanics and Engineering Science and School of Mathematical Sciences, Fudan University, \\ Shanghai 200433, People's Republic of China \\ ${ }^{2}$ LHD, Institute of Mechanics, Chinese Academy of Sciences, Beijing 100190, People's Republic of China
}

\begin{abstract}
SUMMARY
On the basis of the projection method, a higher order compact finite difference algorithm, which possesses a good spatial behavior, is developed for solving the 2D unsteady incompressible Navier-Stokes equations in primitive variable. The present method is established on a staggered grid system and is at least third-order accurate in space. A third-order accurate upwind compact difference approximation is used to discretize the non-linear convective terms, a fourth-order symmetrical compact difference approximation is used to discretize the viscous terms, and a fourth-order compact difference approximation on a cell-centered mesh is used to discretize the first derivatives in the continuity equation. The pressure Poisson equation is approximated using a fourth-order compact difference scheme constructed currently on the nine-point 2D stencil. New fourth-order compact difference schemes for explicit computing of the pressure gradient are also developed on the nine-point 2D stencil. For the assessment of the effectiveness and accuracy of the method, particularly its spatial behavior, a problem with analytical solution and another one with a steep gradient are numerically solved. Finally, steady and unsteady solutions for the lid-driven cavity flow are also used to assess the efficiency of this algorithm. Copyright (c) 2011 John Wiley \& Sons, Ltd.
\end{abstract}

Received 31 October 2009; Revised 23 November 2010; Accepted 8 February 2011

KEY WORDS: Navier-Stokes equations; higher order; compact finite difference; primitive variable; projection method

\section{INTRODUCTION}

The numerical solution of the unsteady incompressible Navier-Stokes (N-S) equations in primitive variable form represents a difficult computational challenge. One major problem comes from the pressure term in the momentum equations, which couples the momentum equations and must be implicitly updated for the incompressibility to be satisfied. The projection method is a widely used technique for solving viscous incompressible flow based on the primitive variable formulations. This technique introduced originally and analyzed by Chorin $[1,2]$ is actually a fractional step method or operator-splitting discretization scheme, in which the computations of the velocity and the pressure are decoupled through a two-step predictor-corrector procedure. In the past several decades, many improved projection methods were developed by some authors [3-9]. In the first step, an intermediate velocity field is computed by solving the momentum equations ignoring the pressure term and the incompressibility constraint. In the second step, according to Helmholtz-Hodge decomposition theorem [10], the intermediate velocity is projected to the space

\footnotetext{
${ }^{*}$ Correspondence to: Zhenfu Tian, Department of Mechanics and Engineering Science, Fudan University, Shanghai 200433, People's Republic of China.

†E-mail: zftian@nxu.edu.cn
} 
of the divergence-free vector fields to get the pressure and the corrected velocity that satisfies the incompressibility condition.

For many application problems it is desirable to use higher order numerical methods to obtain accurate solution. Compact finite difference (FD) methods feature high order accuracy and smaller stencils. Recently, there has been a renewed interest in the development and application of compact FD methods for the numerical solution of the convection-diffusion and N-S equations [11-18]. It is evident that they are not only accurate and effective but also can obtain satisfactory results with a smaller grid mesh.

This paper is primarily aimed at developing a higher order compact FD algorithm, which possesses a good spatial behavior for solving $2 \mathrm{D}$ unsteady incompressible N-S equations in primary variable form based on the projection method. The method is constructed on a staggered grid system and has at least third-order accuracy in spatial dimension. A third-order upwind compact difference approximation proposed in [15] is used to discretize the non-linear convection terms, a fourth-order symmetrical compact difference approximation is used to discretize the viscous terms, and a fourth-order compact difference approximation on a cell-centered mesh is used to discretize the first derivatives in the continuity equation. Especially, a new fourth-order compact difference scheme for approximating the pressure Poisson equation and new explicit schemes for solving the pressure gradient are proposed on the nine-point 2D stencil. Numerical experiments are performed for the validation of accuracy and efficiency of the present algorithm.

\section{MATHEMATICAL MODEL}

The time-dependent incompressible $\mathrm{N}-\mathrm{S}$ equations in primitive variable form can be written as

$$
\begin{aligned}
\mathbf{V}_{t}+(\mathbf{V} \cdot \nabla) \mathbf{V}+\nabla p & =\frac{1}{R e} \nabla^{2} \mathbf{V} \\
\nabla \cdot \mathbf{V} & =0
\end{aligned}
$$

where $p$ is the pressure, $\mathbf{V}=(u, v)$ represents the velocity vector, and $R e$ is Reynolds number.

Most numerical methods for solving Equations (1) and (2) in terms of the primitive variable use a fractional step approach. An approximation to momentum equation (1) is first made to determine a provisional velocity field, and then an elliptic equation is solved that enforces the solenoidal constraint (2) and determines the pressure.

According to Helmholtz-Hodge decomposition theorem [10], an intermediate velocity $\mathbf{V}^{*}$ is calculated by neglecting the contribution of the pressure gradient term. In this step, the explicit Euler scheme is used:

$$
\frac{\mathbf{V}^{*}-\mathbf{V}^{n}}{\triangle t}=\frac{1}{R e} \nabla^{2} \mathbf{V}^{n}-\left(\mathbf{V}^{n} \cdot \nabla\right) \mathbf{V}^{n}
$$

Then, the intermediate velocity $\mathbf{V}^{*}$ can be decomposed into the sum of two vectors:

$$
\mathbf{V}^{*}=\mathbf{V}^{n+1} \oplus \nabla \phi
$$

where vector $\mathbf{V}^{n+1}$ with zero divergence denotes the velocity fields at the next time level, vector $\nabla \phi$ with zero curl. And then discretizing equation (1) and applying (3), we have

$$
\begin{aligned}
\frac{\mathbf{V}^{n+1}-\mathbf{V}^{*}}{\Delta t} & =-\nabla p^{n+1} \\
\nabla \cdot \mathbf{V}^{n+1} & =0
\end{aligned}
$$

The pressure field $p^{n+1}$ is obtained by taking the divergence of formula (5) and applying formula (6)

$$
\nabla^{2} p^{n+1}=\frac{1}{\Delta t} \nabla \cdot \mathbf{V}^{*}
$$


where $p$ denotes a pseudo-pressure when used in a semi-implicit formulation. After solving the pressure and pressure gradient, we can obtain the velocity fields at time level $n+1$ from Equation (5).

\section{HIGHER ORDER COMPACT SCHEMES OF MATHEMATICAL MODEL}

The staggered mesh grid point system is given in Figure 1. We approximate the problem on uniform spatial grids with step $\Delta x$ in $x$-direction and $\Delta y$ in $y$-direction. We denote by $f_{i, j}$ the approximation of a function $f$ at the mesh points $\left(x_{i}=i \triangle x, y_{j}=j \Delta y\right)$. In addition, some difference operators used in this paper are defined as follows:

$$
\begin{aligned}
\delta_{x}^{0} f_{i, j} & =\frac{f_{i+\frac{1}{2}, j}-f_{i-\frac{1}{2}, j}}{\triangle x} \\
\delta_{y}^{0} f_{i, j} & =\frac{f_{i, j+\frac{1}{2}}-f_{i, j-\frac{1}{2}}}{\Delta y} \\
\triangle_{x}^{0} f_{i, j} & =\frac{f_{i+1, j}-f_{i-1, j}}{2 \triangle x} \\
\triangle_{y}^{0} f_{i, j} & =\frac{f_{i, j+1}-f_{i, j-1}}{2 \triangle y} \\
\triangle_{x x} f_{i, j} & =\frac{f_{i+1, j}-2 f_{i, j}+f_{i-1, j}}{\triangle x^{2}} \\
\triangle_{y y} f_{i, j} & =\frac{f_{i, j+1}-2 f_{i, j}+f_{i, j-1}}{\triangle y^{2}}
\end{aligned}
$$

The first momentum equation is approximated at point $\left(i+\frac{1}{2}, j\right)$, the second one at the point $\left(i, j+\frac{1}{2}\right)$. The continuity equation and pressure are approximated at the point $(i, j)$.

\subsection{Discretization of the intermediate velocity}

The convective terms in Equation (3) are discretized by using a third-order accurate upwind compact FD approximation [15]. For example, the term $u \partial u / \partial x$ is split as follows:

$$
u \frac{\partial u}{\partial x}=u^{+} \frac{\partial u}{\partial x}+u^{-} \frac{\partial u}{\partial x}
$$

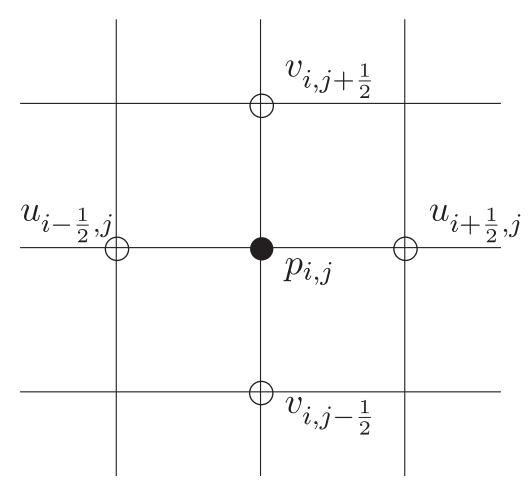

Figure 1. The staggered grid system. 
where $u^{ \pm}=(u \pm|u|) / 2$. The terms $u^{+} \partial u / \partial x$ and $u^{-} \partial u / \partial x$ are approximated at point $\left(i+\frac{1}{2}, j\right)$ by $u_{i+\frac{1}{2}, j}^{+} F_{i+\frac{1}{2}, j}^{+}$and $u_{i+\frac{1}{2}, j}^{-} F_{i+\frac{1}{2}, j}^{-}$, respectively. Here $F_{i+\frac{1}{2}, j}^{+}$and $F_{i+\frac{1}{2}, j}^{-}$can be approximated by the following FD schemes:

$$
\begin{aligned}
& F_{i-\frac{1}{2}, j}^{+}+2 F_{i+\frac{1}{2}, j}^{+}=\frac{1}{2 \triangle x}\left(-5 u_{i-\frac{1}{2}, j}+4 u_{i+\frac{1}{2}, j}+u_{i+\frac{3}{2}, j}\right) \\
& 2 F_{i+\frac{1}{2}, j}^{-}+F_{i+\frac{3}{2}, j}^{-}=\frac{1}{2 \triangle x}\left(-u_{i-\frac{1}{2}, j}-4 u_{i+\frac{1}{2}, j}+5 u_{i+\frac{3}{2}, j}\right)
\end{aligned}
$$

The viscous terms are evaluated by using a fourth-order accurate symmetrical compact FD formulation. For example, the term $\partial^{2} u / \partial x^{2}$ at point $\left(i+\frac{1}{2}, j\right)$ is approximated with $S_{i+\frac{1}{2}, j}$ and

$$
\frac{1}{12} S_{i-\frac{1}{2}, j}+\frac{5}{6} S_{i+\frac{1}{2}, j}+\frac{1}{12} S_{i+\frac{3}{2}, j}=\triangle_{x x} u_{i+\frac{1}{2}, j}
$$

To obtain $S_{i+\frac{1}{2}, j}$, a linear system of equation with a tri-diagonal matrix has to be solved.

In the same way, we can approximate other convection terms and viscous terms in momentum equations. After all convection and viscous terms are evaluated, we can calculate the intermediate velocity $\mathbf{V}^{*}$ through

$$
\begin{aligned}
& \left(u^{*}\right)_{i+\frac{1}{2}, j}=\left(u^{n}+\triangle t G\right)_{i+\frac{1}{2}, j} \\
& \left(v^{*}\right)_{i, j+\frac{1}{2}}=\left(v^{n}+\triangle t H\right)_{i, j+\frac{1}{2}}
\end{aligned}
$$

where

$$
\begin{aligned}
& G=\frac{1}{R e}\left(S_{i}(u)+S_{j}(u)\right)-u^{-} F_{i}^{-}(u)-u^{+} F_{i}^{+}(u)-v^{-} F_{j}^{-}(u)-v^{+} F_{j}^{+}(u) \\
& H=\frac{1}{R e}\left(S_{i}(v)+S_{j}(v)\right)-u^{-} F_{i}^{-}(v)-u^{+} F_{i}^{+}(v)-v^{-} F_{j}^{-}(v)-v^{+} F_{j}^{+}(v)
\end{aligned}
$$

where $u^{ \pm} F_{i}^{ \pm}(g)$ and $v^{ \pm} F_{j}^{ \pm}(g)$ are approximations of $u^{ \pm}(\partial g / \partial x)_{i+\frac{1}{2}, j}$ and $v^{ \pm}(\partial g / \partial y)_{i, j+\frac{1}{2}}$, and $S_{i}(g)$ and $S_{j}(g)$ are approximations of $\left(\partial^{2} g / \partial x^{2}\right)_{i+\frac{1}{2}, j}$ and $\left(\partial^{2} g / \partial y^{2}\right)_{i, j+\frac{1}{2}}$, respectively, and $u, v$ in $G, H$ denote the velocity at time level $n$.

In Equation (21), the value of velocity component $v$, which is defined at point $\left(i, j+\frac{1}{2}\right)$, needs to be computed at the point $\left(i+\frac{1}{2}, j\right)$. In this paper, $v_{i+\frac{1}{2}, j}$ is calculated by fourth-order accurate midpoint interpolation. In the same way, $u_{i, j+\frac{1}{2}}$ can be calculated.

\subsection{Discretization of the pressure field and the pressure gradient}

The Poisson equation for pressure can be written as

$$
\nabla^{2} p=\frac{1}{\triangle t} D\left(\mathbf{V}^{*}\right)
$$

where $D\left(\mathbf{V}^{*}\right)=\left(\partial u^{*} / \partial x\right)+\left(\partial v^{*} / \partial y\right), u^{*}, v^{*}$ are the intermediate velocities. At point $(i, j)$, Equation (22) is discretized with a fourth-order difference approximation on the nine-point 2D stencil, which can be expressed as follows:

$$
\begin{aligned}
& \frac{1}{12 \Delta x \Delta y}\left\{(\lambda+\gamma)\left(p_{i+1, j+1}+p_{i+1, j-1}+p_{i-1, j+1}+p_{i-1, j-1}-20 p_{i, j}\right)\right. \\
& \left.\quad+2(5 \lambda-\gamma)\left(p_{i+1, j}+p_{i-1, j}\right)+2(5 \gamma-\lambda)\left(p_{i, j+1}+p_{i, j-1}\right)\right\} \\
& =\frac{1}{\Delta t}\left\{D_{i, j}+\frac{1}{12}\left[\Delta x^{2}\left(D_{x x}\right)_{i, j}+\Delta y^{2}\left(D_{y y}\right)_{i, j}\right]\right\}
\end{aligned}
$$


where $\lambda=\Delta x / \Delta y, \gamma=\Delta y / \Delta x$, and

$$
\begin{aligned}
D_{i, j} & =\delta_{x} u_{i, j}^{*}+\delta_{y} v_{i, j}^{*} \\
\delta_{x} u_{i, j}^{*} & =\frac{1}{24 \triangle x}\left[27\left(u_{i+\frac{1}{2}, j}^{*}-u_{i-\frac{1}{2}, j}^{*}\right)-\left(u_{i+\frac{3}{2}, j}^{*}-u_{i-\frac{3}{2}, j}^{*}\right)\right] \\
\delta_{y} v_{i, j}^{*} & =\frac{1}{24 \triangle y}\left[27\left(v_{i, j+\frac{1}{2}}^{*}-v_{i, j-\frac{1}{2}}^{*}\right)-\left(v_{i, j+\frac{3}{2}}^{*}-v_{i, j-\frac{3}{2}}^{*}\right)\right]
\end{aligned}
$$

Equations (25) and (26) are fourth-order accurate [13]. For a fully fourth-order FD of Equation (23) we need to approximate $\left(D_{x x}\right)_{i, j}$ and $\left(D_{y y}\right)_{i, j}$ with second-order accuracy, which is done as follows:

$$
\begin{aligned}
\left(D_{x x}\right)_{i, j} & =\left[\left(\frac{\partial u^{*}}{\partial x}+\frac{\partial v^{*}}{\partial y}\right)_{x x}\right]_{i, j} \\
& =\left(\frac{\partial^{3} u^{*}}{\partial x^{3}}+\frac{\partial^{3} v^{*}}{\partial x^{2} \partial y}\right)_{i, j} \\
\left(D_{y y}\right)_{i, j} & =\left(\frac{\partial^{3} u^{*}}{\partial x \partial y^{2}}+\frac{\partial^{3} v^{*}}{\partial y^{3}}\right)_{i, j}
\end{aligned}
$$

Note that

$$
\begin{aligned}
& \left(\frac{\partial^{3} u^{*}}{\partial x^{3}}\right)_{i, j}=\triangle_{x x} \delta_{x}^{0} u_{i, j}^{*}+O\left(\triangle x^{2}\right) \\
& \left(\frac{\partial^{3} v^{*}}{\partial y^{3}}\right)_{i, j}=\triangle_{y y} \delta_{y}^{0} v_{i, j}^{*}+O\left(\triangle y^{2}\right)
\end{aligned}
$$

and

$$
\begin{aligned}
\left(\frac{\partial^{3} v^{*}}{\partial x^{2} \partial y}\right)_{i, j} & =\frac{1}{\Delta y}\left[\left(\frac{\partial^{2} v^{*}}{\partial x^{2}}\right)_{i, j+\frac{1}{2}}-\left(\frac{\partial^{2} v^{*}}{\partial x^{2}}\right)_{i, j-\frac{1}{2}}\right]+O\left(\triangle y^{2}\right) \\
& =\frac{1}{\triangle y}\left(\triangle_{x x} v_{i, j+\frac{1}{2}}^{*}-\triangle_{x x} v_{i, j-\frac{1}{2}}^{*}\right)+O\left(\triangle x^{2}+\triangle y^{2}\right) \\
& =\triangle_{x x} \delta_{y}^{0} v_{i, j}^{*}+O\left(\triangle x^{2}+\triangle y^{2}\right) \\
\left(\frac{\partial^{3} u^{*}}{\partial x \partial y^{2}}\right)_{i, j} & =\triangle_{y y} \delta_{x}^{0} u_{i, j}^{*}+O\left(\triangle x^{2}+\triangle y^{2}\right)
\end{aligned}
$$

we have

$$
\begin{aligned}
& \left(D_{x x}\right)_{i, j}=\triangle_{x x}\left[\delta_{x}^{0} u_{i, j}^{*}+\delta_{y}^{0} v_{i, j}^{*}\right]+O\left(\triangle x^{2}+\triangle y^{2}\right) \\
& \left(D_{y y}\right)_{i, j}=\triangle_{y y}\left[\delta_{y}^{0} v_{i, j}^{*}+\delta_{x}^{0} u_{i, j}^{*}\right]+O\left(\triangle x^{2}+\triangle y^{2}\right)
\end{aligned}
$$

Substituting Equations (24), (25), (26), (33), and (34) into the right-hand side of Equation (23), we obtain the following fourth-order FD scheme for the pressure Poisson equation (22) on staggered 
grid system:

$$
\begin{aligned}
& \frac{1}{12 \Delta x \Delta y}\left[(\lambda+\gamma)\left(p_{i+1, j+1}+p_{i+1, j-1}+p_{i-1, j+1}+p_{i-1, j-1}-20 p_{i, j}\right)\right. \\
& \left.\quad+2(5 \lambda-\gamma)\left(p_{i+1, j}+p_{i-1, j}\right)+2(5 \gamma-\lambda)\left(p_{i, j+1}+p_{i, j-1}\right)\right] \\
& =\frac{1}{12 \Delta t \triangle x}\left\{\frac{1}{2}\left[27\left(u_{i+\frac{1}{2}, j}^{*}-u_{i-\frac{1}{2}, j}^{*}\right)-\left(u_{i+\frac{3}{2}, j}^{*}-u_{i-\frac{3}{2}, j}^{*}\right)\right]+(\Delta x)^{3} \triangle_{x x}\left[\delta_{x}^{0} u_{i, j}^{*}+\delta_{y}^{0} v_{i, j}^{*}\right]\right\} \\
& \quad+\frac{1}{12 \Delta t \Delta y}\left\{\frac{1}{2}\left[27\left(v_{i, j+\frac{1}{2}}^{*}-v_{i, j-\frac{1}{2}}^{*}\right)-\left(v_{i, j+\frac{3}{2}}^{*}-v_{i, j-\frac{3}{2}}^{*}\right)\right]+(\Delta y)^{3} \triangle_{y y}\left[\delta_{y}^{0} v_{i, j}^{*}+\delta_{x}^{0} u_{i, j}^{*}\right]\right\}
\end{aligned}
$$

For computing velocity field, we must know the value of pressure gradient at the same grid point. As noted above, velocity components $u$ and $v$ are defined at points $\left(i+\frac{1}{2}, j\right)$ and $\left(i, j+\frac{1}{2}\right)$ on staggered mesh grid system, respectively. So the pressure gradient components $\partial p / \partial x$ and $\partial p / \partial y$ should be calculated at points $\left(i+\frac{1}{2}, j\right)$ and $\left(i, j+\frac{1}{2}\right)$. In the following text, we give the derivation of FD schemes to approximate the $(\partial p / \partial x)_{i+\frac{1}{2}, j}$ and $(\partial p / \partial y)_{i, j+\frac{1}{2}}$. For $(\partial p / \partial x)_{i+\frac{1}{2}, j}$, using Taylor expansions of $p_{i+1, j}$ and $p_{i, j}$ to point $\left(i+\frac{1}{2}, j\right)$, we have

$$
\left(\frac{\partial p}{\partial x}\right)_{i+\frac{1}{2}, j}=\delta_{x}^{0} p_{i+\frac{1}{2}, j}-\frac{\triangle x^{2}}{24}\left(\frac{\partial^{3} p}{\partial x^{3}}\right)_{i+\frac{1}{2}, j}+O\left(\triangle x^{4}\right)
$$

Note that

$$
\frac{\partial^{2} p}{\partial x^{2}}+\frac{\partial^{2} p}{\partial y^{2}}=\frac{1}{\triangle t} D\left(\mathbf{V}^{*}\right)
$$

and

$$
D\left(\mathbf{V}^{*}\right)=\frac{\partial u^{*}}{\partial x}+\frac{\partial v^{*}}{\partial y}
$$

Combining Equations (37) and (38), and substituting into the second term of the right-hand side of Equation (36) and rearranging, we obtain the following fourth-order compact scheme to approximate the pressure gradient $(\partial p / \partial x)_{i+\frac{1}{2}, j}$

$$
\begin{aligned}
\left(\frac{\partial p}{\partial x}\right)_{i+\frac{1}{2}, j}= & \delta_{x}^{0} p_{i+\frac{1}{2}, j}+\frac{\triangle x^{2}}{24}\left(\frac{\partial^{3} p}{\partial x \partial y^{2}}\right)_{i+\frac{1}{2}, j}-\frac{\triangle x^{2}}{24 \triangle t}\left(\frac{\partial^{2} u^{*}}{\partial x^{2}}+\frac{\partial^{2} v^{*}}{\partial x \partial y}\right)_{i+\frac{1}{2}, j}+O\left(\triangle x^{4}\right) \\
= & \delta_{x}^{0} p_{i+\frac{1}{2}, j}+\frac{\triangle x^{2}}{24} \triangle_{y y} \delta_{x}^{0} p_{i+\frac{1}{2}, j}-\frac{\triangle x^{2}}{24 \triangle t}\left[\triangle_{x x} u_{i+\frac{1}{2}, j}^{*}+\delta_{y}^{0} \delta_{x}^{0} v_{i+\frac{1}{2}, j}^{*}\right] \\
& +O\left(\triangle x^{4}+\triangle x^{2} \triangle y^{2}\right)
\end{aligned}
$$

Similarly, we can obtain the fourth-order accurate compact difference approximation of $(\partial p / \partial y)_{i, j+\frac{1}{2}}$

$$
\begin{aligned}
\left(\frac{\partial p}{\partial y}\right)_{i, j+\frac{1}{2}}= & \delta_{y}^{0} p_{i, j+\frac{1}{2}}+\frac{\triangle y^{2}}{24} \triangle_{x x} \delta_{y}^{0} p_{i, j+\frac{1}{2}}-\frac{\triangle y^{2}}{24 \triangle t}\left[\triangle_{y y} v_{i, j+\frac{1}{2}}^{*}+\delta_{x}^{0} \delta_{y}^{0} u_{i, j+\frac{1}{2}}^{*}\right] \\
& +O\left(\triangle x^{2} \triangle y^{2}+\triangle y^{4}\right)
\end{aligned}
$$




\subsection{Solution of Poisson equation for pressure}

The solution of the pressure Poisson equation (35) will cost the maximum of the whole numerical calculating time, so people are trying to develop an efficient algorithm. In this paper we have designed a line iterative approach with an alternating direction implicit (ADI) procedure that enables us to obtain the solutions of the problems by application of the one-dimensional tridiagonal Thomas algorithm with a considerable saving in computing time.

First, rewriting the discrete pressure equation in the following:

$$
\begin{aligned}
& 2(5 \lambda-\gamma) p_{i-1, j}^{*}-20(\lambda+\gamma) p_{i, j}^{*}+2(5 \lambda-\gamma) p_{i+1, j}^{*}=S_{i, j} \\
& 2(5 \gamma-\lambda) p_{i, j-1}-20(\lambda+\gamma) p_{i, j}+2(5 \gamma-\lambda) p_{i, j+1}=S_{i, j}^{\prime}
\end{aligned}
$$

where

$$
\begin{aligned}
S_{i, j}= & -(\lambda+\gamma)\left(p_{i+1, j+1}+p_{i+1, j-1}+p_{i-1, j+1}+p_{i-1, j-1}\right)-2(5 \gamma-\lambda)\left(p_{i, j+1}+p_{i, j-1}\right) \\
& +\frac{\triangle y}{\triangle t}\left\{\frac{1}{2}\left[27\left(u_{i+\frac{1}{2}, j}^{*}-u_{i-\frac{1}{2}, j}^{*}\right)-\left(u_{i+\frac{3}{2}, j}^{*}-u_{i-\frac{3}{2}, j}^{*}\right)\right]+(\Delta x)^{3} \triangle_{x x}\left[\delta_{x}^{0} u_{i, j}^{*}+\delta_{y}^{0} v_{i, j}^{*}\right]\right\} \\
& +\frac{\triangle x}{\triangle t}\left\{\frac{1}{2}\left[27\left(v_{i, j+\frac{1}{2}}^{*}-v_{i, j-\frac{1}{2}}^{*}\right)-\left(v_{i, j+\frac{3}{2}}^{*}-v_{i, j-\frac{3}{2}}^{*}\right)\right]+(\triangle y)^{3} \triangle_{y y}\left[\delta_{y}^{0} v_{i, j}^{*}+\delta_{x}^{0} u_{i, j}^{*}\right]\right\} \\
S_{i, j}^{\prime}= & -(\lambda+\gamma)\left(p_{i+1, j+1}^{*}+p_{i+1, j-1}^{*}+p_{i-1, j+1}^{*}+p_{i-1, j-1}^{*}\right)-2(5 \lambda-\gamma)\left(p_{i+1, j}^{*}+p_{i-1, j}^{*}\right) \\
& +\frac{\triangle y}{\triangle t}\left\{\frac{1}{2}\left[27\left(u_{i+\frac{1}{2}, j}^{*}-u_{i-\frac{1}{2}, j}^{*}\right)-\left(u_{i+\frac{3}{2}, j}^{*}-u_{i-\frac{3}{2}, j}^{*}\right)\right]+(\triangle x)^{3} \triangle_{x x}\left[\delta_{x}^{0} u_{i, j}^{*}+\delta_{y}^{0} v_{i, j}^{*}\right]\right\} \\
& +\frac{\triangle x}{\triangle t}\left\{\frac{1}{2}\left[27\left(v_{i, j+\frac{1}{2}}^{*}-v_{i, j-\frac{1}{2}}^{*}\right)-\left(v_{i, j+\frac{3}{2}}^{*}-v_{i, j-\frac{3}{2}}^{*}\right)\right]+(\triangle y)^{3} \triangle_{y y}\left[\delta_{y}^{0} v_{i, j}^{*}+\delta_{x}^{0} u_{i, j}^{*}\right]\right\}
\end{aligned}
$$

The first formula of (41) is the implicit iteration of (22) in $x$-direction, the second one in $y$-direction. These two formulas should be iterated alternately in each time step. For periodical boundary problems, the main works are to solve the two linear systems with circulant coefficient matrix, which concerns about CTDMA (cyclic triple diagonal matrix algorithm). For non-periodical boundary problems, it solves mainly the two linear systems with triple diagonal coefficient matrix, which concerns about TDMA (triple diagonal matrix algorithm).

\subsection{Boundary formulation for velocity and pressure}

In order to match high order accuracy at boundary conditions for velocity and pressure, the high order accuracy boundary schemes are designed. Various biased FD schemes are designed for approximation to the first and second derivatives at boundary in this paper. For example, the first derivative at the boundary $j=1$ satisfies the following formulation:

$$
f_{1}^{\prime}+\alpha f_{2}^{\prime}=\frac{1}{\Delta x} \sum_{i=1}^{k} a_{i} f_{i} \quad(k=1,2, \ldots, N)
$$

The coefficients $\alpha$ and $a_{i}$ can be determined by applying Taylor expansion and coupling to necessary of inner points scheme and accuracy. In this paper, the following biased schemes can be obtained, and some relative schemes also can be found in [19].

$$
a_{1}=-\frac{3+\alpha+2 a_{4}}{2}, \quad a_{2}=2+3 a_{4}, \quad a_{3}=-\frac{1-\alpha+6 a_{4}}{2}
$$


where $\alpha, a_{4}$ are free parameters, the scheme has at least second accuracy. Similarly, the following third-order and fourth-order schemes can be obtained as

$$
\begin{gathered}
a_{1}=-\frac{11+2 \alpha}{6}, \quad a_{2}=\frac{6-\alpha}{2}, \quad a_{3}=\frac{2 \alpha-3}{2}, \quad a_{4}=\frac{2-\alpha}{6} \quad \text { (third order) } \\
\alpha=3, \quad a_{1}=-\frac{17}{6}, \quad a_{2}=\frac{3}{2}, \quad a_{3}=\frac{3}{2}, \quad a_{4}=-\frac{1}{6} \quad \text { (fourth order) }
\end{gathered}
$$

In Equation (46), set $\alpha=0$, the third-order explicit biased scheme is defined by

$$
f_{1}^{\prime}=\frac{1}{6 \triangle x}\left(-11 f_{1}+18 f_{2}-9 f_{3}+2 f_{4}\right)
$$

In Equation (45), set $\alpha=2, a_{4}=0$, the third-order implicit biased scheme is defined by

$$
f_{1}^{\prime}+2 f_{2}^{\prime}=\frac{1}{2 \triangle x}\left(-5 f_{1}+4 f_{2}+f_{3}\right)
$$

With the same method, the second derivative at the boundary $j=1$ satisfies the following formulation:

$$
f_{1}^{\prime \prime}+\beta f_{2}^{\prime \prime}=\frac{1}{\triangle x^{2}} \sum_{i=1}^{k} b_{i} f_{i} \quad(k=1,2, \ldots, N)
$$

From Equation (50), the following second-order and third-order biased schemes with free parameters can be defined as

$$
\begin{array}{ll}
b_{1}=\beta+2+b_{5}, & b_{2}=-\left(2 \beta+5+4 b_{5}\right), \quad b_{3}=\beta+4+6 b_{5}, \quad b_{4}=-\left(1+4 b_{5}\right) \\
b_{1}=\frac{11 \beta+35}{12}, & b_{2}=-\frac{5 \beta+26}{3}, \quad b_{3}=\frac{\beta+19}{2}, \quad b_{4}=\frac{\beta-14}{3}, \quad b_{5}=\frac{11-\beta}{12}
\end{array}
$$

Some widely used schemes are obtained from Equations (51) and (52)

$$
\begin{aligned}
& \beta=11, \quad b_{1}=13, \quad b_{2}=-27, \quad b_{3}=15, \quad b_{4}=-1, \quad b_{5}=0 \quad \text { (third order) } \\
& \beta=10, \quad b_{1}=\frac{145}{12}, \quad b_{2}=-\frac{76}{3}, \quad b_{3}=\frac{29}{2}, \quad b_{4}=-\frac{4}{3}, \quad b_{5}=\frac{1}{12} \quad \text { (fourth order) } \\
& \beta=0, \quad b_{1}=2, \quad b_{2}=-5, \quad b_{3}=4, \quad b_{4}=-1, \quad b_{5}=0 \quad \text { (second order) } \\
& \beta=0, \quad b_{1}=\frac{35}{12}, \quad b_{2}=-\frac{26}{3}, \quad b_{3}=\frac{19}{2}, \quad b_{4}=-\frac{14}{3}, \quad b_{5}=\frac{11}{12} \quad \text { (third order) }
\end{aligned}
$$

the higher order boundary scheme can be defined by adding more stencil points in Equations (44) and (50). The schemes (49) and (53) are implicit ones that can couple to inner scheme (15), (16) to use.

Refer to Equation (44), the first derivative of pressure at boundary $i=1$ can be written as $(\alpha=0)$

$$
\left(\frac{\partial p}{\partial x}\right)_{1, j}=\frac{1}{\triangle x} \sum_{i=1}^{k} a_{i} p_{i, j}(k=1,2, \ldots, N)
$$

Then the boundary value of pressure at $i=1$ can be described as

$$
p_{1, j}=\frac{1}{a_{1}}\left[\triangle x\left(\frac{\partial p}{\partial x}\right)_{1, j}-\sum_{i=2}^{k} a_{i} p_{i, j}\right]
$$


It is easy to compute $(\partial p / \partial x)_{1, j}$ by projecting momentum equation to boundary normal direction. If $k=3$ is set in Equation (58) and coupled to Equation (41), the value of inner points can be calculated by using $(\partial p / \partial x)_{1, j}$ and without considering the boundary value. After finishing, to compute the value of inner points, the boundary value can be obtained from Equation (57).

In this subsection, all the boundary schemes are designed for the left boundary; the other boundary schemes can be designed similarly.

\subsection{FD algorithm}

The FD algorithm based on staggered grid system is now briefly outlined.

1. The first step: compute intermediate velocity $\mathbf{V}^{*}$

(a) Applying upwind compact schemes (15) and (16) to compute convective terms and symmetrical compact scheme (17) to compute viscous terms;

(b) Applying (18) and (19) to compute intermediate velocity $\mathbf{V}^{*}$.

2. The second step: projection step

(a) Applying symmetrical compact scheme (35) to compute pressure $p$;

(b) Applying schemes (39), (40) to compute pressure gradient;

(c) Applying scheme (5) to compute new velocity field at time level $n+1$.

3. Repeat process 1-2.

\section{NUMERICAL EXAMPLES}

In this section, we perform numerical experiments to illustrate the accuracy and effectiveness of the proposed higher order compact difference algorithm. The numerical results of three test problems are given. All results were run on a SONY PCG-V505MCP computer using double precision arithmetic.

Example 1

Consider N-S equations (1) and (2) with the initial condition [1]

$$
\begin{aligned}
& u(x, y, 0)=-\cos (N x) \sin (N y) \\
& v(x, y, 0)=\sin (N x) \cos (N y)
\end{aligned}
$$

where $0 \leqslant x \leqslant 2 \pi, 0 \leqslant y \leqslant 2 \pi$. The exact solution for this problem is given by

$$
\begin{aligned}
& u(x, y, t)=-\cos (N x) \sin (N y) \exp \left(-2 N^{2} t / R e\right) \\
& v(x, y, t)=\sin (N x) \cos (N y) \exp \left(-2 N^{2} t / R e\right) \\
& p(x, y, t)=-\frac{1}{4}(\cos (2 N x)+\cos (2 N y)) \exp \left(-4 N^{2} t / R e\right)
\end{aligned}
$$

where $N$ is an integer.

The numerical solutions are obtained under uniform grids $(h=\Delta x=\Delta y)$ with different mesh sizes and a fixed time step size $(\triangle t=0.002)$ and compared under the RMS (root mean square) errors of the numerical solution with respect to the exact solution. The RMS error is estimated by

$$
\operatorname{err}_{\mathrm{RMS}}=\sqrt{\frac{1}{N_{x} \times N_{y}} \sum\left(\phi_{i, j_{\mathrm{comput}}}-\phi_{i, j_{\mathrm{exact}}}\right)^{2}}
$$

where $N_{x}$ and $N_{y}$ are grid points in $x$ - and $y$-directions, respectively, and $\phi$ stands for $u, v, p$, and $\psi$. 
Table I. RMS errors with the rate of convergence at $t=2$ for $u, v, \psi, p(\operatorname{Re}=100, N=2)$.

\begin{tabular}{lcccccccc}
\hline Grid & $u$-error & Rate & $v$-error & Rate & $\psi$-error & Rate & $p$-error & Rate \\
\hline $11 \times 11$ & $7.0070489(-2)$ & - & $7.0070489(-2)$ & - & $3.0677609(-1)$ & - & $1.0764149(-1)$ & - \\
$21 \times 21$ & $9.0692193(-3)$ & 3.16 & $9.0692193(-3)$ & 3.16 & $3.8426509(-2)$ & 3.21 & $1.0567607(-2)$ & 3.58 \\
$31 \times 31$ & $2.8851487(-3)$ & 2.94 & $2.8851487(-3)$ & 2.94 & $1.1948521(-2)$ & 3.00 & $2.9103288(-3)$ & 3.31 \\
$41 \times 41$ & $1.2238736(-3)$ & 3.06 & $1.2238736(-3)$ & 3.06 & $5.0439820(-3)$ & 3.08 & $1.1356134(-3)$ & 3.36 \\
$51 \times 51$ & $6.3063026(-4)$ & 3.03 & $6.3063026(-4)$ & 3.03 & $2.5915402(-3)$ & 3.05 & $5.3933641(-4)$ & 3.41 \\
\hline
\end{tabular}

Note: $7.0070489(-2)=7.0070489^{-2}$, etc.

Table II. Errors with the rate of convergence at $t=2$ for $p$ and CPU times $(R e=100, N=2)$.

\begin{tabular}{lccccccc}
\hline & \multicolumn{3}{c}{ Second-order method } & & \multicolumn{3}{c}{ Present method } \\
\cline { 2 - 5 } \cline { 7 - 8 } Grid size & $L^{2}$-error & Rate & CPU times & & $L^{2}$-error & Rate & CPU times \\
\hline $11 \times 11$ & $1.405 \mathrm{E}-01$ & & 0.281 & & $1.076 \mathrm{E}-01$ & & 0.344 \\
$21 \times 21$ & $4.160 \mathrm{E}-02$ & 1.882 & 0.640 & & $1.057 \mathrm{E}-02$ & 3.588 & 0.875 \\
$31 \times 31$ & $1.897 \mathrm{E}-02$ & 2.016 & 1.984 & & $2.910 \mathrm{E}-03$ & 3.312 & 2.625 \\
$41 \times 41$ & $1.073 \mathrm{E}-02$ & 2.037 & 5.156 & & $1.136 \mathrm{E}-03$ & 3.364 & 6.266 \\
$51 \times 51$ & $6.869 \mathrm{E}-03$ & 2.044 & 10.141 & & $5.393 \mathrm{E}-04$ & 3.413 & 12.015 \\
$61 \times 61$ & $4.759 \mathrm{E}-03$ & 2.050 & 19.422 & & $2.817 \mathrm{E}-04$ & 3.627 & 22.375 \\
$71 \times 71$ & $3.483 \mathrm{E}-03$ & 2.056 & 33.265 & & $1.554 \mathrm{E}-04$ & 3.918 & 37.172 \\
$81 \times 81$ & $2.654 \mathrm{E}-03$ & 2.063 & 55.594 & & $8.488 \mathrm{E}-05$ & 4.590 & 60.640 \\
\hline
\end{tabular}

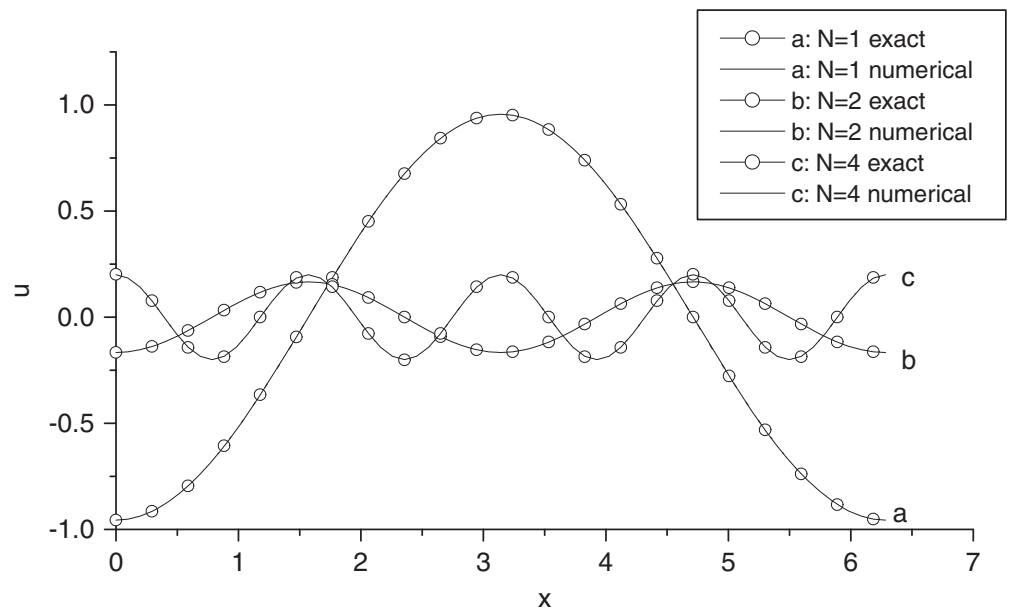

Figure 2. Velocity component $u$ at $J=16$ for $N=1,2,4$ and $R e=100$.

The comparison of numerical results is listed in Tables I and II. The rate of convergence is estimated for velocity, vorticity, and pressure by using

$$
\text { rate }=-\frac{\log (\operatorname{err} 1 / \text { err } 2)}{\log \left(N_{1} / N_{2}\right)}
$$

where err 1 and err 2 are RMS errors with the grid system having $\left(N_{1}+1\right) \times\left(N_{1}+1\right)$ and $\left(N_{2}+\right.$ 1) $\times\left(N_{2}+1\right)$ points, respectively.

It clearly shows from Table I that these values are almost 3, i.e. the present algorithm is third-order accurate in space. Table II shows the comparisons of the grid size and the CPU time required to achieve similar accuracy by both the present method and the second-order method 
Table III. Grid dependence of $u$-velocity at the middle vertical line.

\begin{tabular}{lrrrrr}
\hline$\frac{y}{2 \pi}$ & $33 \times 33$ & $65 \times 65$ & $97 \times 97$ & $129 \times 129$ & $161 \times 161$ \\
\hline$t=6$ & & & & & \\
0.125 & -1.184842 & -1.179558 & -1.193791 & -1.195409 & -1.195281 \\
0.375 & 1.118550 & 1.174393 & 1.190816 & 1.193237 & 1.193570 \\
0.625 & 0.721181 & 0.675312 & 0.671645 & 0.671016 & 0.670807 \\
0.875 & -0.721712 & -0.675761 & -0.671872 & -0.671161 & -0.670914 \\
$t=8$ & & & & & \\
0.125 & -1.212727 & -1.248724 & -1.239336 & -1.229696 & -1.222598 \\
0.375 & 1.205786 & 1.241997 & 1.235062 & 1.227654 & 1.222152 \\
0.625 & 0.601131 & 0.569668 & 0.565301 & 0.564269 & 0.563725 \\
0.875 & -0.589285 & -0.557180 & -0.556850 & -0.557907 & -0.558628 \\
$t=10$ & & & & & \\
0.125 & -1.114304 & -1.190062 & -1.218252 & -1.220469 & -1.218174 \\
0.375 & 1.108267 & 1.187827 & 1.216600 & 1.219821 & 1.218185 \\
0.625 & 0.479549 & 0.319745 & 0.312882 & 0.313072 & 0.313879 \\
0.875 & -0.511503 & -0.388838 & -0.372990 & -0.365419 & -0.360031 \\
\hline
\end{tabular}

Table IV. Grid dependence of $v$-velocity at the middle horizontal line.

\begin{tabular}{lrrrrr}
\hline$\frac{x}{2 \pi}$ & $33 \times 33$ & $65 \times 65$ & $97 \times 97$ & $129 \times 129$ & $161 \times 161$ \\
\hline$t=6$ & & & & & \\
0.125 & 0.528987 & 0.573284 & 0.577703 & 0.579244 & 0.580072 \\
0.375 & 0.286628 & 0.273020 & 0.268318 & 0.266371 & 0.265384 \\
0.625 & -0.563078 & -0.588918 & -0.588034 & -0.586974 & -0.586250 \\
0.875 & -0.248347 & -0.256254 & -0.257363 & -0.258304 & -0.258944 \\
$t=8$ & & & & & \\
0.125 & 0.626262 & 0.688566 & 0.697838 & 0.700261 & 0.701517 \\
0.375 & 0.430775 & 0.467724 & 0.470392 & 0.470401 & 0.470019 \\
0.625 & -0.677859 & -0.709125 & -0.712632 & -0.711363 & -0.710431 \\
0.875 & -0.408829 & -0.448764 & -0.457935 & -0.461006 & -0.462497 \\
$t=10$ & & & & & \\
0.125 & 0.661511 & 0.670398 & 0.675042 & 0.679404 & 0.682410 \\
0.375 & 0.554838 & 0.589328 & 0.580261 & 0.578208 & 0.577004 \\
0.625 & -0.633699 & -0.644967 & -0.649541 & -0.656733 & -0.662788 \\
0.875 & -0.499663 & -0.558352 & -0.562290 & -0.564907 & -0.566180 \\
\hline
\end{tabular}

(i.e. the convective and viscous terms in the momentum equations and the pressure Poisson equation are approximated by using the standard second-order central formula). Note that the computed solutions from the present method are more accurate than that from the secondorder method at the same mesh size. It is clear that the second-order method requires almost the same CPU time as does the present method for the same mesh size. It is also clear that the second-order method requires a much finer grid, hence much longer CPU time, to achieve similar accuracy as the present method. For example, in the case of $41 \times 41$ grid points, the present method requires only $6.266 \mathrm{~s}$ to achieve an accuracy of $1.136 \mathrm{e}-03$, whereas the secondorder method requires a grid of $81 \times 81$ and $55.594 \mathrm{~s}$ of CPU time to achieve an accuracy of $2.654 \mathrm{e}-03$. The results in Table II show the superiority of the present method over the second-order method.

In Figure 2, the variation of the exact and numerical horizontal velocities $u$ with $x$ at $y=\pi / 2$ for $N=1,2,4$ is given. One can hardly distinguish the numerical solutions from the analytical ones. This figure shows again that the present method can give the satisfying result for this problem. 
Table V. Percentage difference of $u$-velocity at the middle vertical line with respect to the results on a gird of $161 \times 161$.

\begin{tabular}{lcccc}
\hline$\frac{y}{2 \pi}$ & $33 \times 33$ & $65 \times 65$ & $97 \times 97$ & $129 \times 129$ \\
\hline$t=6$ & & & & \\
0.125 & 0.873 & 1.315 & 0.125 & 0.011 \\
0.375 & 6.285 & 1.607 & 0.231 & 0.028 \\
0.625 & 7.509 & 0.672 & 0.125 & 0.031 \\
0.875 & 7.571 & 0.722 & 0.143 & 0.037 \\
$t=8$ & & & & 0.581 \\
0.125 & 0.807 & 2.137 & 1.369 & 0.450 \\
0.375 & 1.339 & 1.624 & 1.056 & 0.097 \\
0.625 & 6.635 & 1.054 & 0.280 & \\
0.875 & 5.488 & 0.259 & 0.318 & 0.188 \\
$t=10$ & & & & 0.134 \\
0.125 & 8.527 & 2.308 & 0.006 & 0.257 \\
0.375 & 9.023 & 2.492 & 0.130 & 1.497 \\
0.625 & 52.782 & 1.869 & 0.318 & \\
0.875 & 42.072 & 8.001 & 3.600 & \\
\hline
\end{tabular}

Table VI. Percentage difference of $v$-velocity at the middle horizontal line with respect to the results on a gird of $161 \times 161$.

\begin{tabular}{lrrrr}
\hline$\frac{x}{2 \pi}$ & $33 \times 33$ & $65 \times 65$ & $97 \times 97$ & $129 \times 129$ \\
\hline$t=6$ & & & & \\
0.125 & 8.807 & 1.170 & 0.408 & 0.143 \\
0.375 & 8.005 & 2.877 & 1.105 & 0.372 \\
0.625 & 3.952 & 0.455 & 0.304 & 0.124 \\
0.875 & 4.092 & 1.039 & 0.611 & 0.247 \\
$t=8$ & & & & \\
0.125 & 10.727 & 1.846 & 0.525 & 0.179 \\
0.375 & 8.349 & 0.488 & 0.079 & 0.081 \\
0.625 & 4.585 & 0.184 & 0.310 & 0.131 \\
0.875 & 11.604 & 2.969 & 0.986 & \\
$t=10$ & & & & 0.440 \\
0.125 & 3.063 & 1.760 & 1.080 & 0.209 \\
0.375 & 3.842 & 2.136 & 0.564 & 0.914 \\
0.625 & 4.389 & 2.689 & 1.999 & 0.225 \\
0.875 & 11.748 & 1.383 & 0.687 & \\
\hline
\end{tabular}

\section{Example 2}

The next problem is set up to test the behavior of the discretization for non-stationary situations when steep gradients are involved. This is a doubly periodic double shear layer flow problem taken from [3], the initial conditions are taken as

$$
\begin{aligned}
& u(x, y, 0)= \begin{cases}\tanh [(y-\pi / 2) / \rho] & 0 \leqslant y \leqslant \pi \\
\tanh [(3 \pi / 2-y) / \rho] & \pi \leqslant y \leqslant 2 \pi\end{cases} \\
& v(x, y, 0)=\sigma \sin (x)
\end{aligned}
$$

where $\rho=\pi / 15$ is the thickness of shear layer, $\sigma=0.05$ is the initial perturbation which will grow with time and leads to a roll up of the shear layer. This problem, which has the characteristic of large gradient for $R e=10000$, is an excellent case to verify the precision and stability of the numerical scheme at high wavenumbers. 


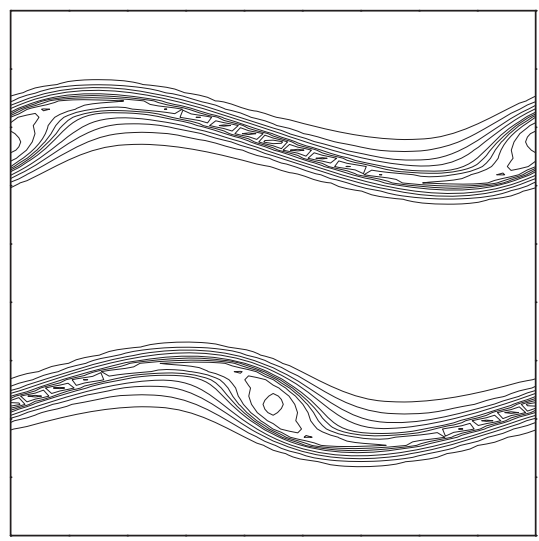

(a)

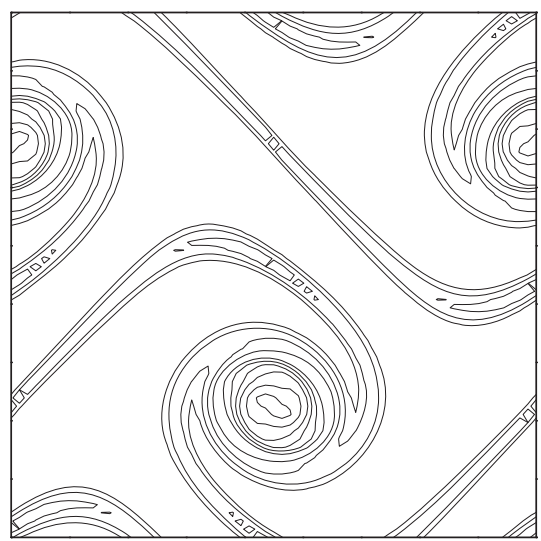

(c)

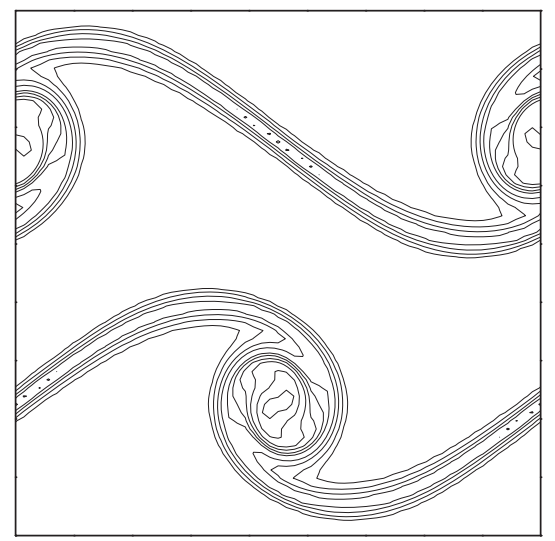

(b)

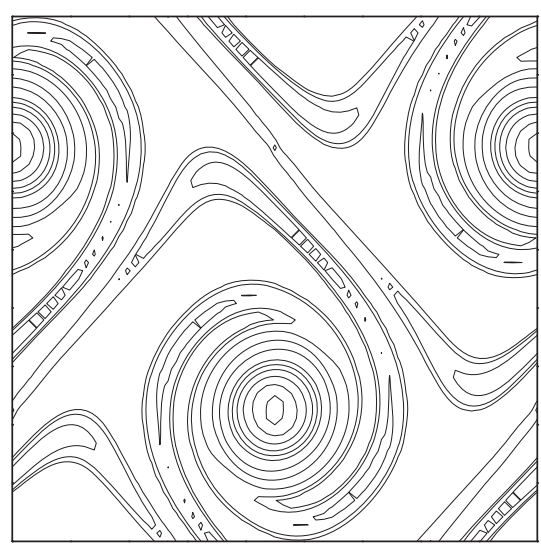

(d)

Figure 3. Vorticity contours for $\triangle x=\Delta y=2 \pi / 64$ : (a) $t=4$; (b) $t=6$; (c) $t=8$; and (d) $t=10$.

The incompressible N-S equations (1) and (2) with periodic boundary conditions are solved by using the present method. The calculated values of horizontal velocity $u$ along the vertical centerline and the calculated values of vertical velocity $v$ along the horizontal centerline at $t=6,8$ and 10 are listed in Tables III and IV for the grids of $33 \times 33,65 \times 65,97 \times 97,129 \times 129$, and $161 \times 161$. Based on the finest grid $(161 \times 161)$ solutions, the deviations of the results on the relatively coarser grids are shown in Tables V and VI. The solutions on a grid of $129 \times 129$ are very close to that on the finest grid, and the differences are small enough. It is believed that the grid of $129 \times 129$ points is appropriate in the present computations.

Computed vortices at different time levels for $\Delta x=\Delta y=2 \pi / 64$ (i.e. $65 \times 65$ grid points) and $\triangle x=\Delta y=2 \pi / 128$ (i.e. $129 \times 129$ grid points) are given in Figures 3 and 4 , respectively. It is seen that very good resolution is obtained on the medium grids (e.g. $129 \times 129$ grid points). The present results are very smooth and the integration is very stable. Results from Figures 3 and 4 show that the present method is efficient for solving this problem with a steep gradient. For comparison, Figure 5 shows the flows computed at $t=10$ by using the two second-order methods, named as the CDS1 (i.e. the spatial derivatives of velocities are approximated by the second-order difference scheme and the pressure Poisson equation is approximated by the fourth-order difference scheme) and the CDS2 (i.e. the spatial derivatives of all variables are approximated by the second-order difference scheme), respectively. Clearly, the second-order method on a grid of $161 \times 161$ has difficulty in capturing the thin shear layers, resulting in unphysical noise visible in the shear region and from the blurring effect on shear layers in the vortex core. On the contrary, the present method, even in the case of $65 \times 65$ grid points, performs very well and can capture the thin shear layers on a grid of $129 \times 129$ (see Figure 4) requiring 6787.26 s CPU time. Note also that the CDS1 method 


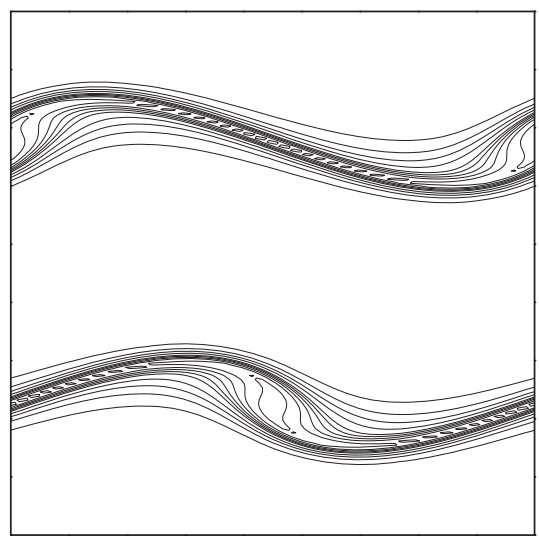

(a)

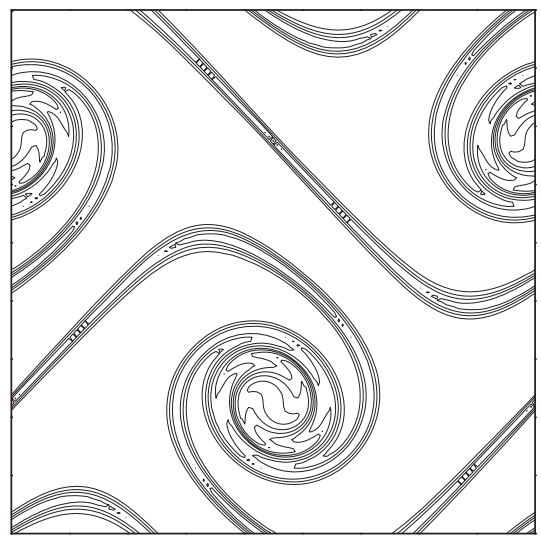

(c)

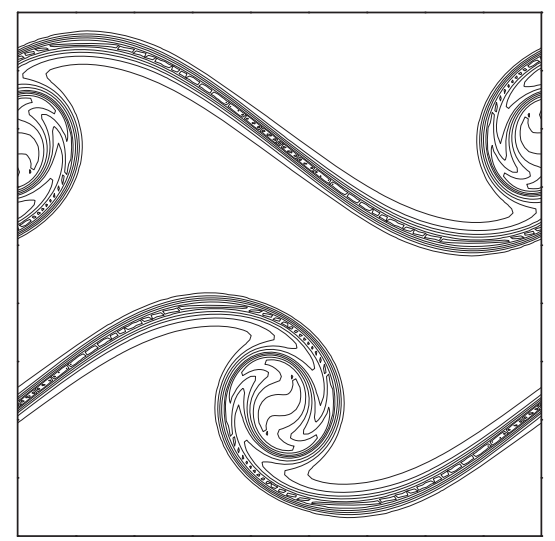

(b)

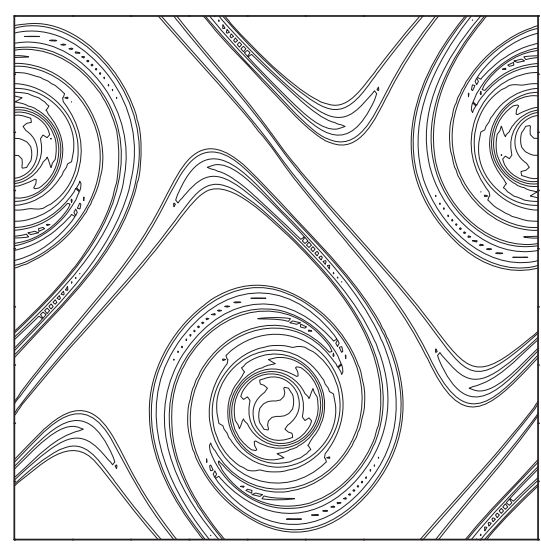

(d)

Figure 4. Vorticity contours for $\Delta x=\Delta y=2 \pi / 128$ : (a) $t=4$; (b) $t=6$; (c) $t=8$; and (d) $t=10$.
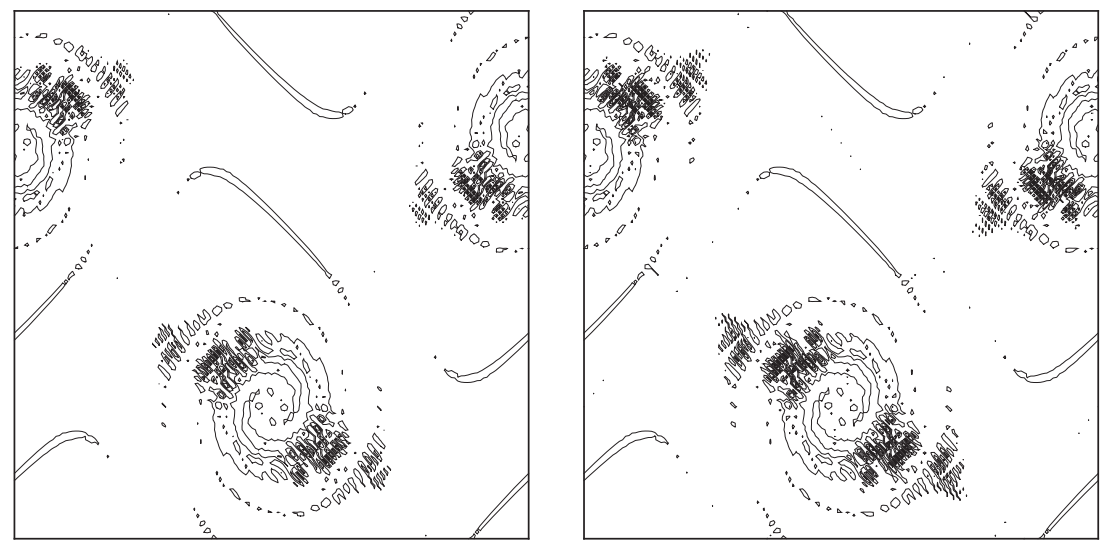

Figure 5. Vortex contours computed at $t=10$ with (left) the second-order scheme for the velocities whereas the fourth-order scheme for the pressure and (right) the second-order scheme for all variables.

and the CDS2 method spend $14169.75 \mathrm{~s}$ and $12359.98 \mathrm{~s}$ CPU time, respectively. The numerical results from this example indicate that the present method is far superior to the second-order methods (i.e. the CDS1 and the CDS2), especially in computational accuracy, efficiency, and resolution. 


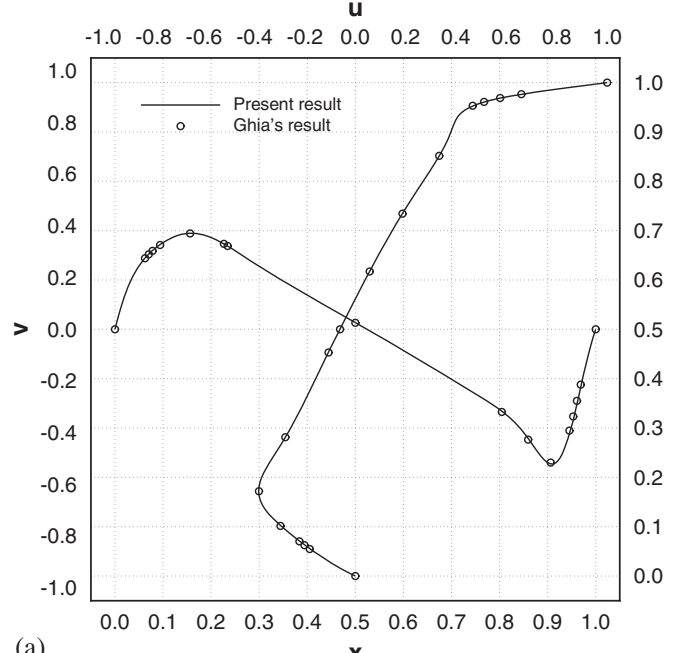

(a)

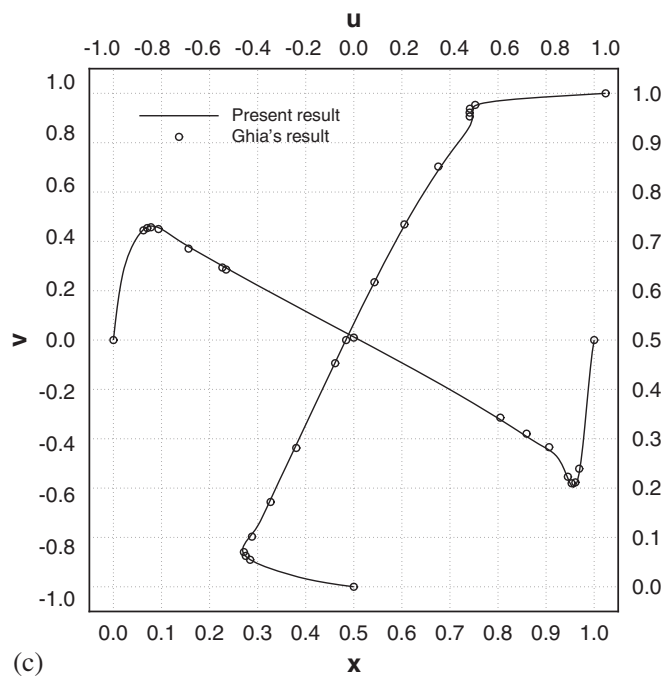

$\mathbf{u}$

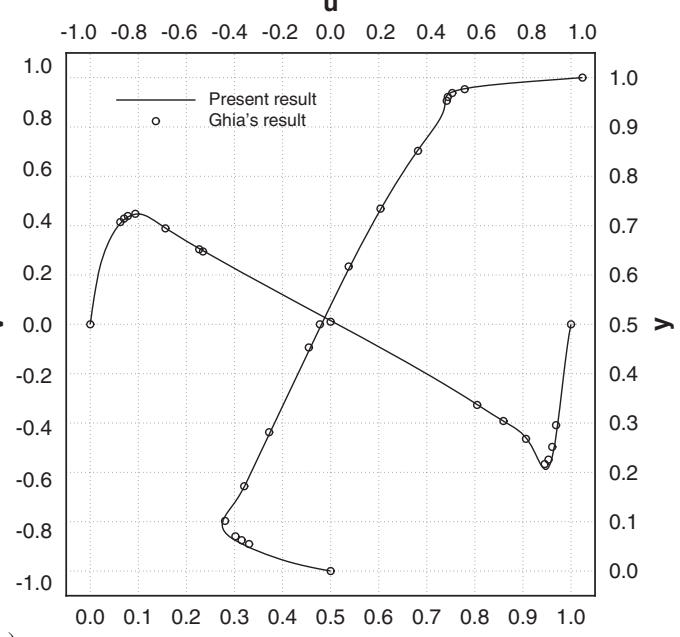

(b)

$\mathbf{x}$

$\mathbf{u}$

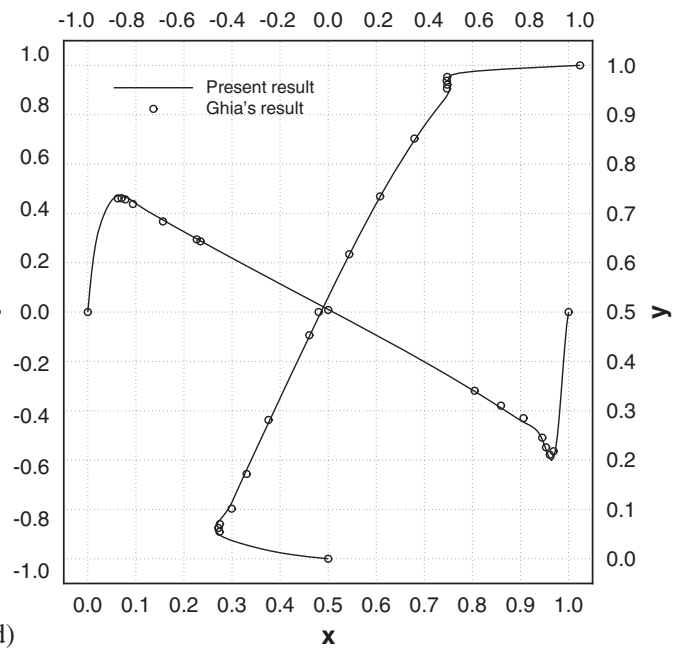

Figure 6. Profiles of $u$-velocity along vertical line and $v$-velocity along horizontal line through geometric center in cavity for (a) $R e=1000$; (b) $R e=3200$; (c) $R e=5000$; and (d) $R e=7500$.

\section{Example 3}

Finally, the two-dimensional lid-driven cavity problem is considered. Over the years, this problem is often used [6,20-26] to demonstrate the accuracy and efficiency of numerical methods for incompressible flows. This problem is of great scientific interest because it displays almost all fluid mechanical phenomena for incompressible viscous flows in the simplest of geometric settings.

Computational region is $0 \leqslant x \leqslant 1$ and $0 \leqslant y \leqslant 1$, the boundary conditions are taken as

$$
\left\{\begin{array}{lll}
u=1 & v=0 & y=1 \\
u=0 & v=0 & x=0, \quad 1 \text { and } y=0
\end{array}\right.
$$

Let

$$
\triangle u_{\max }(n \triangle t)=\max \left[\left(u_{i, j}^{n+1}-u_{i, j}^{n}\right)^{2}+\left(v_{i, j}^{n+1}-v_{i, j}^{n}\right)^{2}\right]^{\frac{1}{2}}
$$

denote the root square error at the $(n+1)$ time level. When $\Delta u_{\max }(n \triangle t) \leqslant 10^{-8}$, the solution is qualified as steady. And in each time level, to obtain the convergent solutions for the pressure, 

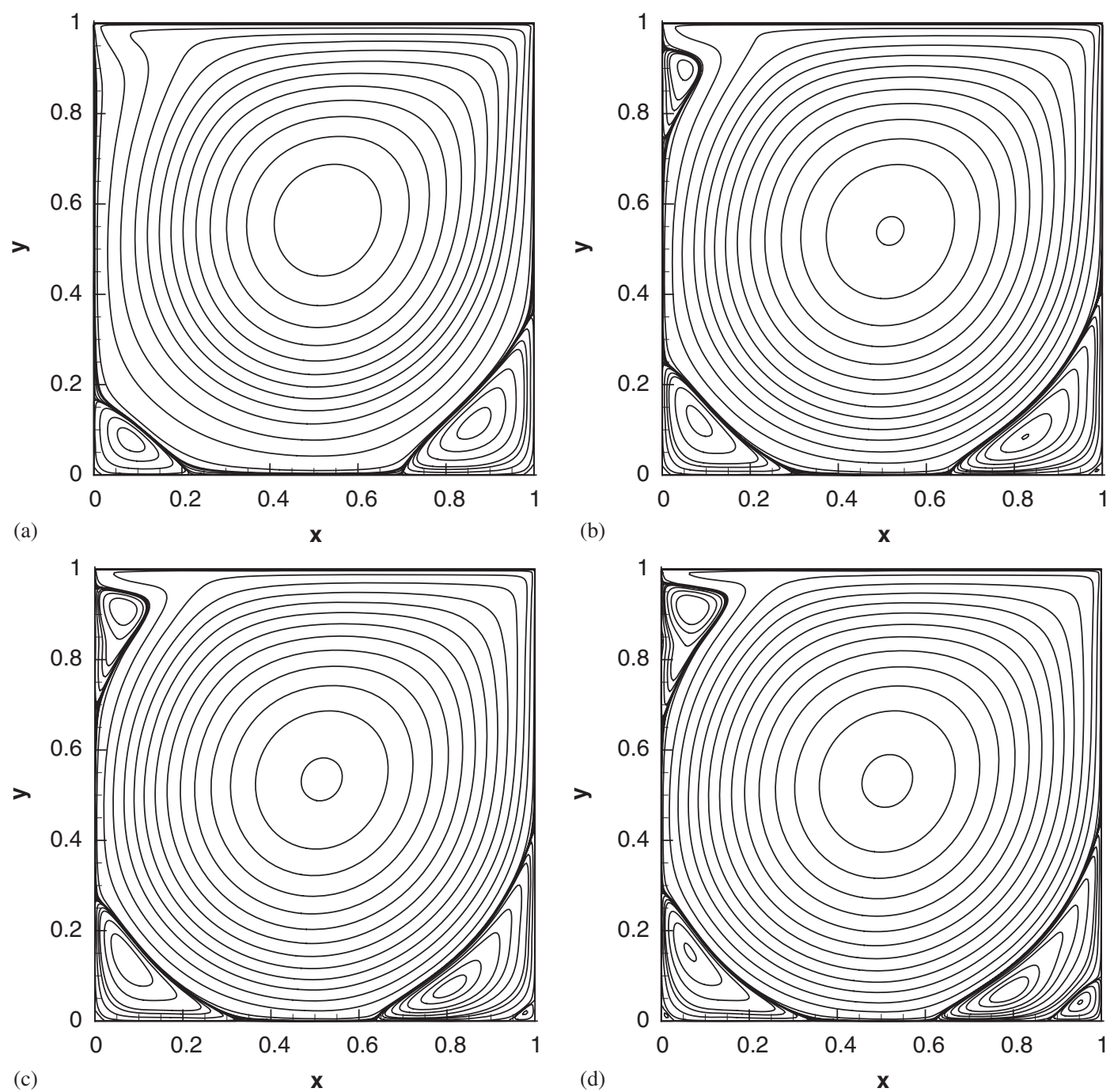

Figure 7. The steady-state streamline contours: (a) $R e=1000$; (b) $R e=3200$; (c) $R e=5000 ;$ and (d) $R e=7500$.

more iterations are used until

$$
\triangle P_{\max }^{m}=\max \left|P_{i, j}^{m+1}-P_{i, j}^{m}\right| \leqslant 10^{-6}
$$

where $m$ denotes the iteration number.

Numerical solutions for the driven-cavity flow are obtained at different Reynolds numbers. In present computation, the grid mesh spacing is equal to $\frac{1}{128}$ and $\frac{1}{256}$ and the time increment is equal to 0.001 .

Comparisons of some characteristic flow variables are made with previous numerical results obtained by Ghia et al. [25] for different values of the Reynolds number, $1000 \leqslant R e \leqslant 7500$. In Figure 6, comparisons of the vertical velocities on the horizontal centerline and the horizontal velocities on the vertical centerline of the square cavity are exhibited for $1000 \leqslant R e \leqslant 7500$ with those of Ghia et al. [25]. Noted that velocity profiles obtained by the proposed method on the grid mesh spacing $\frac{1}{128}$ match very well with those of Ghia et al. [25]. In [25], the computation is carried out on the fine grid mesh spacing $\frac{1}{256}$. 


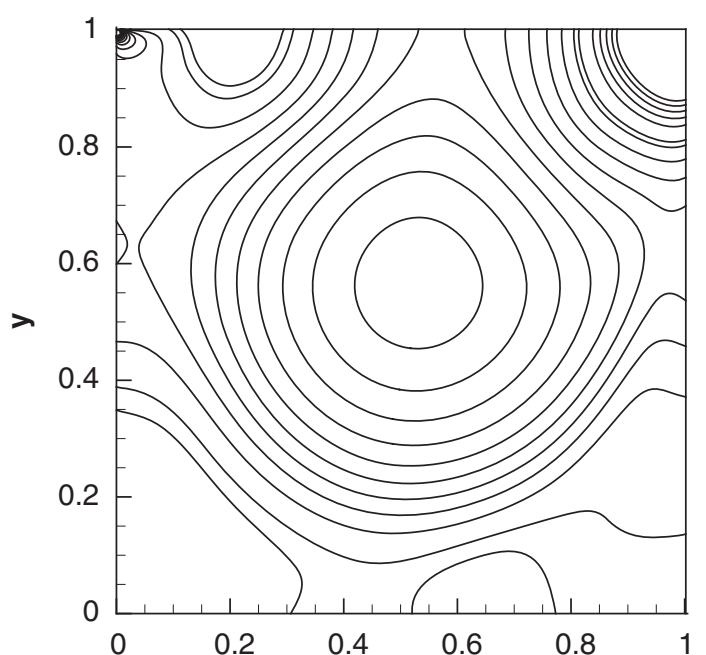

(a)

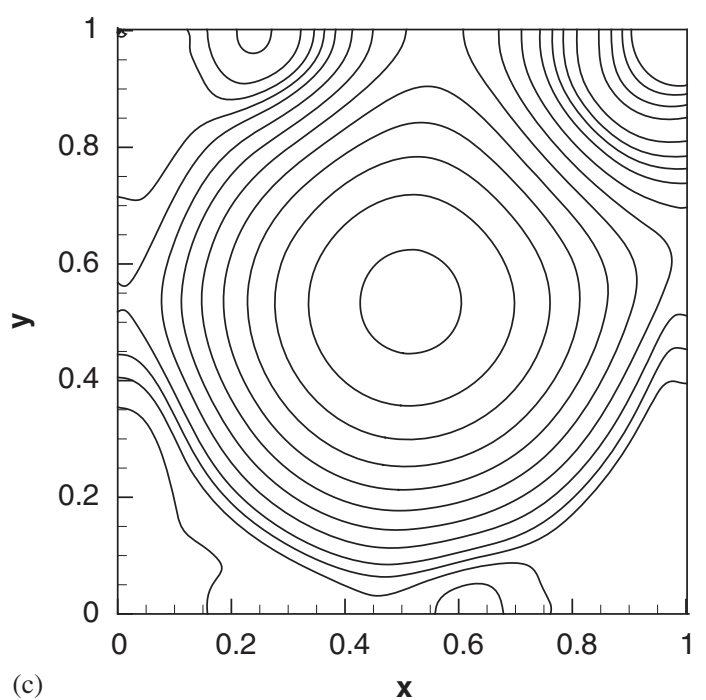

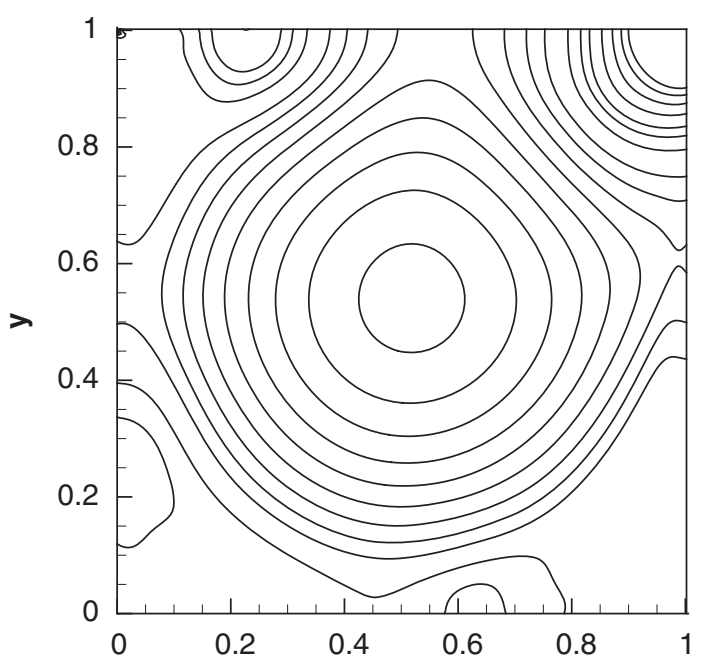

(b)

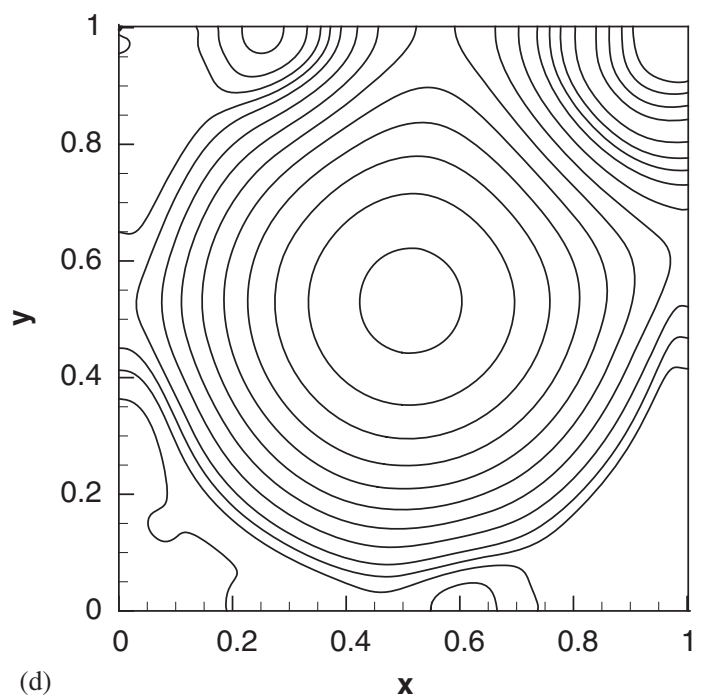

Figure 8. The steady-state pressure contours: (a) $R e=1000$; (b) $R e=3200$; (c) $R e=5000$; and (d) $R e=7500$.

Figure 7 exhibits the streamline contours for the cavity flows with Reynolds numbers 1000 , 3200,5000 , and 7500 . In these graphs, the typical separations and secondary vortices at the bottom corners of the cavity as well as at the top left can be seen. These stream function profiles are in very good agreement with the benchmark results of Ghia et al. [25] and other established results $[6,20,21,24]$ thereby confirming that the present method yields quantitatively accurate solutions. Figure 8 shows pressure contours for $1000 \leqslant R e \leqslant 7500$. Our pressure contours are compared with established results in [20]. Again, a pretty consistent comparison is obtained.

To further validate the present method quantitatively, the minimum values $u_{\min }$ and the corresponding location coordinate $y_{\min }$ of horizontal velocity along the vertical centerline, the maximum values $v_{\max }$ and the corresponding location coordinate $x_{\max }$ of vertical velocity along the horizontal centerline, and the values of streamfunction at the primary vortex center and the corresponding locations obtained numerically are collected and listed in Table VII for different Reynolds numbers $(1000 \leqslant R e \leqslant 7500)$. The available comparison data from the literature are also given in this table. In Table VIII, the location of the center of secondary vortices and the value of streamfunction 
Table VII. The minimum values of the velocity component $u$ along $x=0.5$ and the corresponding $y$-coordinate $y_{\min }$, the maximum values of the velocity component $v$ along $y=0.5$ and the corresponding $x$-coordinate $x_{\max }$, and the stream function $\psi$ and the location coordinates $(x, y)$ of the center of primary vortex for different Reynolds numbers $1000 \leqslant R e \leqslant 7500$.

\begin{tabular}{|c|c|c|c|c|c|c|c|c|}
\hline$R e$ & References & Grid size & $u_{\min }$ & $y_{\min }$ & $v_{\max }$ & $x_{\max }$ & $x$ & $y$ \\
\hline \multirow[t]{5}{*}{1000} & Present & $129 \times 129$ & -0.38134 & 0.1719 & 0.36907 & $0.1563-0.116985$ & 0.5313 & 0.5625 \\
\hline & Present & $257 \times 257$ & -0.38373 & 0.1719 & 0.37181 & $0.1602-0.117610$ & 0.5313 & 0.5625 \\
\hline & Ghia et al. [25] & $129 \times 129$ & -0.38289 & 0.1719 & 0.37095 & $0.1563-0.117929$ & 0.5313 & 0.5625 \\
\hline & $\begin{array}{l}\text { Sahin and } \\
\text { Owens [27] }\end{array}$ & $257 \times 257$ & -0.38810 & 0.1727 & 0.37691 & $0.1573-0.118800$ & 0.5335 & 0.5639 \\
\hline & $\begin{array}{l}\text { Bruneau and } \\
\text { Jouron [21] }\end{array}$ & $257 \times 257$ & -0.37640 & 0.1602 & 0.36650 & $0.1523-0.116300$ & 0.5313 & 0.5586 \\
\hline \multirow[t]{4}{*}{3200} & Present & $129 \times 129$ & -0.42328 & 0.0938 & 0.41825 & $0.1016-0.118514$ & 0.5156 & 0.5391 \\
\hline & Present & $257 x$ & -0.43050 & 0.0938 & 0.42703 & $0.0977-0.120475$ & 0.5195 & 0.5391 \\
\hline & Ghia et al. [25] & $129 \times 129$ & -0.41933 & 0.1016 & 0.42768 & $0.0938-0.120377$ & 0.5156 & 0.5469 \\
\hline & $\begin{array}{l}\text { Sahin and } \\
\text { Owens [27] }\end{array}$ & $257 \times 257$ & -0.43540 & 0.0921 & 0.43245 & $0.0972-0.121628$ & 0.5201 & 0.5376 \\
\hline \multirow[t]{5}{*}{5000} & Present & $129 \times 129$ & -0.43056 & 0.0781 & 0.42814 & $0.0781-0.11 \varepsilon$ & 0.5156 & 0.5313 \\
\hline & Present & $257 \times 257$ & -0.44153 & 0.0742 & 0.44095 & $0.0820-0.121062$ & 0.5156 & 0.5352 \\
\hline & Ghia et al. [25] & $257 \times 257$ & -0.43643 & 0.0703 & 0.43648 & $0.0781-0.118966$ & 0.5117 & 0.5352 \\
\hline & $\begin{array}{l}\text { Sahin and } \\
\text { Owens [27] }\end{array}$ & $257 \times 257$ & -0.44731 & 0.0741 & 0.44691 & $0.0799-0.122050$ & 0.5134 & 0.5376 \\
\hline & $\begin{array}{l}\text { Bruneau and } \\
\text { Jouron [21] }\end{array}$ & $257 \times 257$ & -0.43590 & 0.0664 & 0.42590 & $0.0762-0.114200$ & 0.5156 & 0.5313 \\
\hline \multirow[t]{5}{*}{7500} & Present & $129 \times 129$ & -0.43294 & 0.0625 & 0.43335 & $0.0703-0.117$ & 0.5156 & 0.5313 \\
\hline & Present & $257 \times 257$ & -0.44873 & 0.0625 & 0.45143 & $0.0664-0.121605$ & 0.5117 & 0.5313 \\
\hline & Ghia et al. [25] & $257 \times 257$ & -0.43590 & 0.0625 & 0.44030 & $0.0703-0.119976$ & 0.5117 & 0.5322 \\
\hline & $\begin{array}{l}\text { Sahin and } \\
\text { Owens [27] }\end{array}$ & $257 \times 257$ & -0.45605 & 0.0610 & 0.45805 & $0.0670-0.122302$ & 0.5134 & 0.5289 \\
\hline & $\begin{array}{l}\text { Bruneau and } \\
\text { Jouron [21] }\end{array}$ & $257 \times 257$ & -0.43790 & 0.0508 & 0.41790 & $0.0625-0.111300$ & 0.5156 & 0.5234 \\
\hline
\end{tabular}

at vortex center obtained in the present computation are compared with data from the literature $[20,21,23,25,28,29]$ at Reynolds numbers $1000,3200,5000$, and 7500 . It is clear from all these comparisons that the results of the present numerical method are reliable and the algorithm can be used to solve unsteady viscous incompressible flows.

A further illustration of the accuracy and the efficiency of the present method is given by computation of the unsteady flow at higher $R e=10000$ on the grid mesh spacing $\frac{1}{128}$. Auteri et al. [30] has found that the critical Reynolds number $R e_{c}$ of Hopf bifurcation from stationary state to non-stationary periodic state lies in the interval $8017.6<R e_{c}<8018.8$. Thus for the computations at $R e=10000$ we expect the flow to show unsteady periodic behavior. Starting from steady-state solution obtained at $R e=7500$, the solution reaches its asymptotic periodic state at about $t=1240$ $(t=n \triangle t)$. A series of nine instantaneous iso-streamfunction contours for one complete cycle is displayed in Figure 9. It is shown that the iso-vorticity contours are better resolved through the use of higher order compact schemes for a given fixed grid $129 \times 129$. Note that the computed solution is smooth in most of the remaining domain. The reason for this is that there are sharp gradients close to the upper moving wall. All the features shown by Fu et al. [15] are well represented. As observed by Fu et al., persistent oscillations develop at secondary and tertiary vortices, particularly the appearance and the disappearance of the two tertiary vortices at the bottom and top lift side.

Table IX lists the comparisons of the grid size and the CPU time required to achieve accurate results (with respect to the benchmark solution [25]), at $R e=5000$, by both the present method and the second-order method (CDS1) (i.e. the spatial derivatives of velocities are approximated by the second-order difference scheme and the pressure Poisson equation is approximated by the 
Table VIII. Comparison of the results of secondary vortex at different Reynolds numbers.

\begin{tabular}{|c|c|c|c|c|c|c|c|}
\hline \multirow[b]{2}{*}{ Re } & \multirow[b]{2}{*}{ References } & \multicolumn{3}{|c|}{ Bottom left } & \multicolumn{3}{|c|}{ Bottom right } \\
\hline & & $\psi$ & $x$ & $y$ & $\psi$ & $x$ & $y$ \\
\hline \multirow[t]{7}{*}{1000} & Present $^{1}$ & $2.20 \mathrm{E}-04$ & 0.0859 & 0.0781 & $1.67 \mathrm{E}-03$ & 0.8594 & 0.1094 \\
\hline & Present $^{2}$ & $2.23 \mathrm{E}-04$ & 0.0820 & 0.0781 & $1.69 \mathrm{E}-03$ & 0.8672 & 0.1133 \\
\hline & Ghia et al. [25] & $2.31 \mathrm{E}-04$ & 0.0859 & 0.0781 & $1.75 \mathrm{E}-03$ & 0.8594 & 0.1094 \\
\hline & Schreiber and Keller [28] & $2.17 \mathrm{E}-04$ & 0.0857 & 0.0714 & $1.70 \mathrm{E}-03$ & 0.8643 & 0.1071 \\
\hline & Hou et al. [20] & $2.22 \mathrm{E}-04$ & 0.0902 & 0.0784 & $1.69 \mathrm{E}-03$ & 0.8667 & 0.1137 \\
\hline & Bruneau and Jouron [21] & $3.25 \mathrm{E}-04$ & 0.0859 & 0.0820 & $1.91 \mathrm{E}-03$ & 0.8711 & 0.1094 \\
\hline & Gupta and Kalita [23] & $2.02 \mathrm{E}-03$ & 0.0875 & 0.0750 & $1.70 \mathrm{E}-03$ & 0.8625 & 0.1125 \\
\hline \multirow[t]{4}{*}{3200} & Present $^{1}$ & $1.08 \mathrm{E}-03$ & 0.0781 & 0.1250 & $2.76 \mathrm{E}-03$ & 0.8281 & 0.0859 \\
\hline & Present $^{2}$ & $1.10 \mathrm{E}-03$ & 0.0820 & 0.1172 & $2.79 \mathrm{E}-03$ & 0.8281 & 0.0859 \\
\hline & Ghia et al. [25] & $9.78 \mathrm{E}-04$ & 0.0859 & 0.1094 & $3.14 \mathrm{E}-03$ & 0.8125 & 0.0859 \\
\hline & Gupta and Kalita [23] & $1.03 \mathrm{E}-03$ & 0.0813 & 0.1188 & $2.86 \mathrm{E}-03$ & 0.8125 & 0.0875 \\
\hline \multirow[t]{6}{*}{5000} & Present $^{1}$ & $1.35 \mathrm{E}-03$ & 0.0703 & 0.1406 & $3.01 \mathrm{E}-03$ & 0.8047 & 0.0703 \\
\hline & Present $^{2}$ & $1.36 \mathrm{E}-03$ & 0.0742 & 0.1367 & $3.04 \mathrm{E}-03$ & 0.8086 & 0.0742 \\
\hline & Ghia et al. [25] & $1.36 \mathrm{E}-03$ & 0.0703 & 0.1367 & $3.08 \mathrm{E}-03$ & 0.8056 & 0.0742 \\
\hline & Hou et al. [20] & $1.35 \mathrm{E}-03$ & 0.0784 & 0.1313 & $3.03 \mathrm{E}-03$ & 0.8078 & 0.0745 \\
\hline & Bruneau and Jouron [21] & $2.22 \mathrm{E}-03$ & 0.0664 & 0.1484 & $4.65 \mathrm{E}-03$ & 0.8301 & 0.0703 \\
\hline & Gupta and Kalita [23] & $1.32 \mathrm{E}-03$ & 0.0750 & 0.1313 & $2.96 \mathrm{E}-03$ & 0.8000 & 0.0750 \\
\hline \multirow[t]{6}{*}{7500} & Present $^{1}$ & $1.50 \mathrm{E}-03$ & 0.0625 & 0.1563 & $3.18 \mathrm{E}-03$ & 0.7891 & 0.0625 \\
\hline & Present $^{2}$ & $1.53 \mathrm{E}-03$ & 0.0625 & 0.1563 & $3.22 \mathrm{E}-03$ & 0.7930 & 0.0664 \\
\hline & Ghia et al. [25] & $1.47 \mathrm{E}-03$ & 0.0645 & 0.1504 & $3.28 \mathrm{E}-03$ & 0.7813 & 0.0625 \\
\hline & Hou et al. [20] & $1.45 \mathrm{E}-03$ & 0.0706 & 0.1529 & $3.20 \mathrm{E}-03$ & 0.7922 & 0.0667 \\
\hline & Bruneau and Jouron [21] & $1.76 \mathrm{E}-03$ & 0.0703 & 0.1289 & $8.32 \mathrm{E}-03$ & 0.8828 & 0.0820 \\
\hline & Gupta and Kalita [23] & $1.60 \mathrm{E}-03$ & 0.0688 & 0.1500 & $3.05 \mathrm{E}-03$ & 0.7813 & 0.0625 \\
\hline
\end{tabular}

Notes: 1 . Solved with $129 \times 129$ grid size; 2 . Solved with $257 \times 257$ grid size.

fourth-order difference scheme). It is clear that the computed solutions from the present method are more close to the benchmark solution [25] than those from the second-order method at the same mesh size. Note that from Table IX, in order to obtain excellent agreement with the benchmark solutions, the present method requires a grid of $161 \times 161$ and $17798.249 \mathrm{~s}$ of CPU time, whereas the second-order method (CDS1) requires a grid of $257 \times 257$ and $38114.438 \mathrm{~s}$ of CPU time. The results in Table IX also exhibit the superiority of the present method over the second-order method.

\section{CONCLUSIONS}

In this work we have developed a new high order accurate compact FD projection algorithm for solving the incompressible N-S equations in primitive variables based on the staggered grid system. The present algorithm has at least third-order accuracy in spatial direction. Especially, the fourthorder compact difference scheme is proposed for approximating the pressure Poisson equation. The simple line iterative or line iterative SOR technique with an ADI procedure enables one to deal with only diagonally dominant tri-diagonal systems which can be solved by application of the one-dimensional tridiagonal Thomas algorithm with a considerable saving in computing time. This permits combining the computational efficiency of the lower order methods with superior accuracy inherent in high order approximations. The proposed discretization technique may be applicable to three-dimension case.

Numerical experiments are performed to demonstrate high accuracy and efficiency of the present method. The computational results show that, besides the excellent performances in computational 

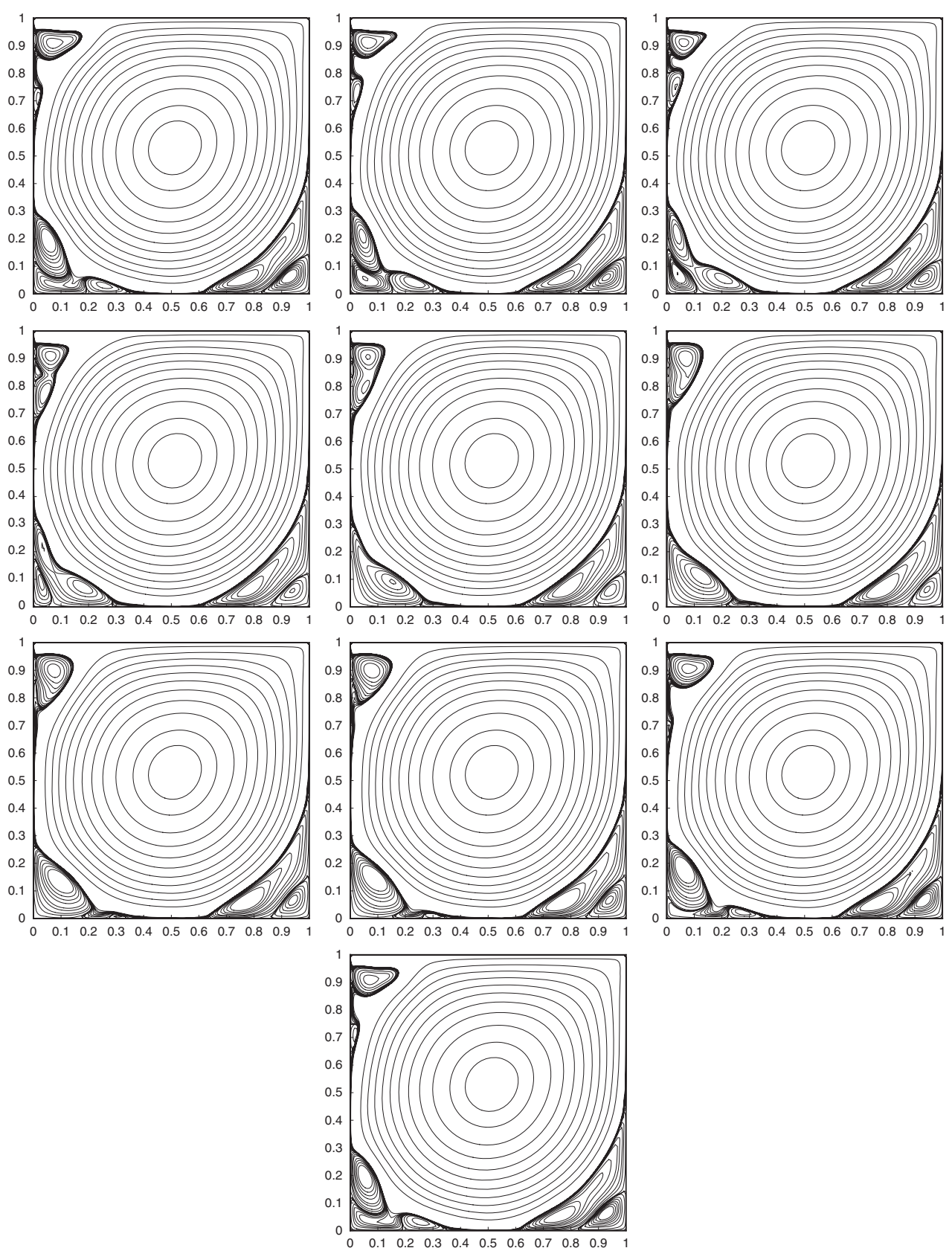

Figure 9. Continuous streamline contours for $R e=10000$.

accuracy, efficiency, and stability, the method has the advantage of better scale resolution with smaller number of grid nodes. It is shown that the current method has large potential of extending to direct numerical simulation of the complex flows, including the flow problems with large gradient and high Reynolds number.

This work may be regarded as a first step towards the establishment of high order accurate and efficient algorithms for the solution of the incompressible N-S equations in primitive variables. Higher order explicit operator-splitting discretizations for temporal variables and high order compact difference schemes with high resolution for spatial variables will be the subject of forthcoming developments. In addition, we mention that the usage of non-uniform mesh becomes an 
Table IX. Comparison of the velocity results of the second-order method (CDS1) with the present third-order method for lid-driven cavity flow at $R e=5000$ and the percentage differences with respect to the benchmark solution [25].

\begin{tabular}{|c|c|c|c|c|c|}
\hline Grid size & $u_{\min }$ & Difference $(\%)$ & $v_{\max }$ & Difference $(\%)$ & CPU time \\
\hline \multicolumn{6}{|c|}{ Second-order method } \\
\hline $128 \times 128$ & -0.40375 & -7.49 & 0.40152 & -8.01 & 6651.400 \\
\hline $160 \times 160$ & -0.41862 & -4.08 & 0.41720 & -4.42 & 9857.816 \\
\hline $192 \times 192$ & -0.42722 & -2.11 & 0.42654 & -2.28 & 17049.468 \\
\hline $256 \times 256$ & -0.43720 & 0.18 & 0.43665 & 0.04 & 38114.438 \\
\hline \multicolumn{6}{|c|}{ Third-order method } \\
\hline $128 \times 128$ & -0.43056 & -1.34 & 0.42184 & -1.91 & 11844.687 \\
\hline $160 \times 160$ & -0.43619 & -0.05 & 0.43463 & -0.42 & 17988.249 \\
\hline $192 \times 192$ & -0.43859 & 0.50 & 0.43754 & 0.24 & 28390.206 \\
\hline $256 \times 256$ & -0.44153 & 1.17 & 0.44095 & 1.02 & 51709.853 \\
\hline
\end{tabular}

essential aspect for dealing with irregular domain problems [31-33]. Based on the approaches proposed in $[31,32]$, the present scheme can be extended to the irregular case.

\section{ACKNOWLEDGEMENTS}

This work was supported in part by the National Natural Science Foundation of China under Grants 10972058 and 10662006, Research Fund for the Doctoral Program of Higher Education of China, the Teaching and Research Award Program for Outstanding Young Teachers in Higher Education Institutions of MOE, People's Republic of China, China Postdoctoral Science Foundation, and the High Performance Computing Foundation of China under Grants 99107 and 00108.

The authors gratefully acknowledge the anonymous referees for their valuable suggestions and comments on the revision of this manuscript.

\section{REFERENCES}

1. Chorin AJ. Numeriacl solution of the Navier-Stokes equations. Mathematics of Computation 1968; 22:745-762.

2. Chorin AJ. On the convergence of discrete approximation to the Navier-Stokes equations. Mathematics of Computation 1969; 23:341-353.

3. Bell JB, Colella P. A second-order projection method for the incompressible Navier-Stokes equations. Journal of Computational Physics 1989; 85:257-283.

4. E W, Liu J-G. Projection method I: convergence and numerical boundary layers. SIAM Journal on Numerical Analysis 1995; 33:1017-1057.

5. E W, Liu J-G. Projection method II: Godunov-Ryabenki analysis. SIAM Journal on Numerical Analysis 1996; 33:1597-1621.

6. Botella O. On the solution of the Navier-Stokes equations using Chebyshev projection schemes with third-order accuracy in time. Computer and Fluids 1997; 26:107-116.

7. Brown DL, Cortez R, Minion ML. Accurate projection methods for the incompressible Navier-Stokes equations. Journal of Computational Physics 2001; 168:464-499.

8. Lopez JM, Marques F, Shen J. An efficient spectral-projection method for the Navier-Stokes equations in cylindrical geometries II. Three-dimensional cases. Journal of Computational Physics 2002; 176:384-401.

9. Auteri F, Parolini N. A mixed-basis spectral projection methods. Journal of Computational Physics 2002; 175:1-23.

10. Ferziger JH, Peric M. Computational Methods for Fluid Dynamics. Springer: Berlin, 1996.

11. Li M, Tang T, Fornberg B. A compact fourth-order finite difference scheme for the incompressible Navier-Stokes equations. International Journal for Numerical Methods in Fluids 1995; 20:1137-1151.

12. Li M, Tang T. A compact fourth-order finite difference scheme for unsteady viscous incompressible flows. Journal of Scientific Computing 2001; 16:29-45.

13. Ma Y, Fu D, Kobarash T, Taniguchi N. Numerical solution of the incompressible Navier-Stokes equations with an upwind compact difference scheme. International Journal for Numerical Methods in Fluids 1999; 30:509-521.

14. Spotz WF, Carey GF. High-order compact scheme for the steady stream-function vorticity equations. International Journal for Numerical Methods in Engineering 1995; 38:3497-3512.

15. Fu D, Ma Y. High resolution scheme. In Computational Fluid Dynamics Review, Hafez M, Oshima K (eds). Wiley: New York, 1995; 234-250. 
16. Sanyasiraju YVSS, Manjula V. Higher order semi compact scheme to solve transient incompressible Navier-Stokes equations. Computational Mechanics 2005; 35:441-448.

17. Tian ZF, Ge YB. A Fourth-order compact finite difference scheme for the steady streamfunction-vorticity formulation of the Navier-Stokes/Boussinesq equations. International Journal for Numerical Methods in Fluids 2003; 41:495-518.

18. Ferreira de Sousa PJSA, Pereira JCF. Fourth- and tenth-order compact finite difference solutions of perturbed circular vortex flows. International Journal for Numerical Methods in Fluids 2005 49:603-618.

19. Lele SK. Compact finite difference schemes with spectral-like resolution. Journal of Computational Physics 1992; 103: $16-42$.

20. Hou S, Zou Q, Chen S, Doolen G, Cogley AC. Simulation of cavity flow by the Lattice Boltzmann method. Journal of Computational Physics 1995; 118:329-347.

21. Bruneau CH, Jouron C. An efficient scheme for solving steady incompressible Navier-Stokes equations. Journal of Computational Physics 1990; 89:389-413.

22. Ben-Artzi M, Croisille J-P, Fishelov D, Trachtenberg S. A pure-compact scheme for the streamfunction formulation of Navier-Stokes equations. Journal of Computational Physics 2005; 205:640-664.

23. Gupta MM, Kalita JC. A new paradigm for solving Navier-Stokes equations: streamfunction-velocity formulation. Journal of Computational Physicis 2005; 207:52-68.

24. Stéphane A, Stéphane V. A 2D compact fourth-order projection decomposition method. Journal of Computational Physics 2005; 206:252-276.

25. Ghia U, Ghia KN, Shin CT. High-Re solutions for incompressible flow using the Navier-Stokes equations and a multigrid method. Journal of Computational Physics 1982; 48:387-411.

26. Deng GB, Piquet J, Queutey P, Visonneau M. Incompressible-flow calculations with a consistent physical interpolations finite-volume approach. Computers and Fluids 1994; 23(8):1029-1047.

27. Sahin M, Owens RG. A novel fully impicit finit volume method applied to the lid-driven cavity problem-Part I: High Reynolds number flow calculations. International Journal for Numerical Methods in Fluids 2003; 42:57-77.

28. Schreiber R, Keller HB. Driven cavity flows by efficient numerical techniques. Journal of Computational Physics 1983; 49:310-333.

29. Kim J, Moin P. Application of a fractional-step method to incompressible Navier-Stokes equations. Journal of Computational Physics 1985; 59:308-323.

30. Auteri F, Parolini N, Quartapelle L. Numerical investigation on the stability of singular driven cavity flow. Journal of Computational Physics 2002; 183:1-25.

31. Gamet L, Ducros F, Nicoud F, Poinsot T. Compact finite difference schemes on non-uniform meshes. Application to direct numerical simulations of compressible flows. International Journal for Numerical Methods in Fluids 1999; 29(2):159-191.

32. Knikker R. Study of a staggered fourth-order compact scheme for unsteady incompressible viscous flows. International Journal for Numerical Methods in Fluids 2009; 59:1063-1092.

33. Ben-Artzi M, Chorev I, Croisille J-P, Fishelov D. A compact difference scheme for the biharmonic equation in planar irregular domains. SIAM Journal of Numerical Analysis 2009; 47:3087-3108. 